\title{
1 CCR2-dependent monocyte-derived cells restrict SARS-CoV-2 infection
}

3 Abigail Vanderheiden ${ }^{1,2,3}$, Jeronay Thomas ${ }^{5,6}$, Allison L. Soung ${ }^{7}$, Meredith E. Davis-Gardner ${ }^{1,2,3}$,

4 Katharine Floyd ${ }^{1,2,3}$, Fengzhi $\mathrm{Jin}^{2,3,4}$, David A. Cowan²,3,10, Kathryn Pellegrini ${ }^{2,3,10}$, Adrian

5 Creanga $^{8}$, Amarendra Pegu $^{8}$, Alexandrine Derrien-Colemyn ${ }^{8}$, Pei-Yong Shi ${ }^{9}$, Arash Grakoui $^{2,3,4}$,

6 Robyn S. Klein ${ }^{7,11}$, Steven E. Bosinger ${ }^{2,3,10}$, Jacob E. Kohlmeier ${ }^{5,6}$, Vineet D. Menachery ${ }^{12}$, Mehul

7 S. Suthar ${ }^{1,2,3,6^{*}}$

$9{ }^{1}$ Center for Childhood Infections and Vaccines of Children's Healthcare of Atlanta, Department of Pediatrics, Emory

10 University School of Medicine, Atlanta, GA, USA.

11 'Emory Vaccine Center, Emory University, Atlanta, GA, USA.

$12{ }^{3}$ Yerkes National Primate Research Center, Atlanta, GA, USA.

$13{ }^{4}$ Division of Infectious Diseases, Emory University School of Medicine, Atlanta, GA, USA.

14 5Department of Microbiology and Immunology, Emory University, Atlanta, GA, USA.

$15{ }^{6}$ Emory-UGA Center of Excellence of Influenza Research and Surveillance (CEIRS), Atlanta GA, USA

$16{ }^{7}$ Center for Neuroimmunology \& Neuroinfectious Diseases, Department of Medicine, Washington University School of

17 Medicine, St. Louis, MO USA.

$18{ }^{8}$ Vaccine Research Center, National Institute of Allergy and Infectious Diseases, National Institutes of Health, Bethesda,

19 MD, USA

$20{ }^{9}$ Department of Biochemistry and Molecular Biology, The University of Texas Medical Branch, Galveston, TX, USA

$21{ }^{10}$ Department of Pathology and Laboratory Medicine, Emory University School of Medicine, Atlanta, GA 30322, USA.

$22{ }^{11}$ Departments of Pathology \& Immunology, Neurosciences, Washington University School of Medicine, St. Louis, MO 23 USA.

$24 \quad{ }^{12}$ Department of Microbiology and Immunology, Institute for Human Infection and Immunity, World Reference Center 25 for Emerging Viruses and Arboviruses, University of Texas Medical Branch, Galveston, TX, USA. 


\section{Abstract}

SARS-CoV-2 has caused a historic pandemic of respiratory disease (COVID-19) and current evidence suggests severe disease is associated with dysregulated immunity within the respiratory tract. However, the innate immune mechanisms that mediate protection during COVID-19 are not

33 well defined. Here we characterize a mouse model of SARS-CoV-2 infection and find that early

34 CCR2-dependent infiltration of monocytes restricts viral burden in the lung. We find that a recently developed mouse-adapted MA-SARS-CoV-2 strain, as well as the emerging B.1.351 variant, trigger an inflammatory response in the lung characterized by expression of pro-inflammatory cytokines and interferon-stimulated genes. scRNA-seq analysis of lung homogenates identified a hyper-inflammatory monocyte profile. Using intravital antibody labeling, we demonstrate that MASARS-CoV-2 infection leads to increases in circulating monocytes and an influx of CD45+ cells into the lung parenchyma that is dominated by monocyte-derived cells. We utilize this model to

41 demonstrate that mechanistically, CCR2 signaling promotes infiltration of classical monocytes

42 into the lung and expansion of monocyte-derived cells. Parenchymal monocyte-derived cells

43 appear to play a protective role against MA-SARS-CoV-2, as mice lacking CCR2 showed higher

44 viral loads in the lungs, increased lung viral dissemination, and elevated inflammatory cytokine

45 responses. These studies have identified a CCR2-monocyte axis that is critical for promoting viral 46 control and restricting inflammation within the respiratory tract during SARS-CoV-2 infection. 
49 Severe acute respiratory syndrome coronavirus 2 (SARS-CoV-2) is a novel $\beta$-coronavirus which

50 emerged in Wuhan, China in December 2019 and is the causative agent of coronavirus disease

512019 (COVID-19) $)^{1,2}$. Innate immunity to SARS-CoV-2 begins with a limited interferon (IFN)

52 response and production of inflammatory cytokines (IL-6, II-1 $\beta$, TNF $\alpha$, IL-8) by respiratory

53 epithelial cells or alveolar macrophages ${ }^{3-6,7}$. As shown in bronchoalveolar lavages (BAL) of

54 COVID-19 patients, this innate immune response coincides with robust infiltration of neutrophils,

55 monocytes, and dendritic cells into the lung airways $s^{6,8}$. Monocytes in the lung parenchyma can be

56 divided into subpopulations characterized by their expression of Ly6C; Ly6C high classical

57 monocytes are pro-inflammatory, whereas Ly6C low non-classical monocytes promote wound

58 healing ${ }^{9,10}$. Ly6C low monocytes are prevalent during homeostatic conditions, however after a

59 viral infection Ly6C high monocytes will infiltrate the lung in a CCR2 dependent manner ${ }^{10-12}$.

60 Classical Ly6C high monocytes can differentiate into monocyte-derived dendritic cells (moDCs),

61 which increase in number in response to viral respiratory infection, produce Type I IFN, and excel

62 at antigen presentation ${ }^{13}$. The contribution of monocytes to promote protective immunity to SARS-

63 CoV-2 infection is not known. In this study, we utilize a mouse-adapted (MA) SARS-CoV-2 strain

64 and the human variant B.1.351 to evaluate the contribution of monocytes to protective immunity

65 against SARS-CoV-2 and identify a CCR2-monocyte axis that is critical for promoting viral control

66 and restricting inflammation within the respiratory tract during SARS-CoV-2 infection.

69 To investigate the immunological response to SARS-CoV-2 in the lung, we generated a MA-

70 SARS-CoV-2 strain (Supplemental Fig. 1A). We engineered mutations into the icSARS-CoV-2

71 backbone ${ }^{14}$ that have been shown to increase SARS-CoV-2 virulence in mice ${ }^{15}$. Next, this virus 
72 was serially passaged 20 times in the lungs of Balb/c mice. Deep sequencing of plaque isolated

73 virus revealed three additional acquired mutations which include two within the Spike (K417N and

$74 \mathrm{H} 655 \mathrm{Y}$ ) and one within the Envelope (E8V) gene. Next, we confirmed the utility of MA-SARS-

75 CoV-2 as a model to study SARS-CoV-2 pathogenesis in C57BI/6 mice. Intranasally infected mice

76 survived infection with MA-SARS-CoV-2, but had $10 \%$ body weight loss at day 2-3 post infection

77 (p.i.) (Fig. 1A). Lung tissue was harvested at 0, 2, and 4 days p.i., and infectious MA-SARS-CoV-

782 was measured via plaque assay. MA-SARS-CoV-2 titers peaked at day 2 p.i, with $10^{9}$ plaque

79 forming units (PFU) per gram of lung tissue. Viral RNA peaked in the lung at day 2 p.i., dropping

80100 -fold by day 4 p.i (Fig. 1B). To determine localization of MA-SARS-CoV-2 in the lung, we

81 performed in situ hybridization using probes that target the Spike gene of SARS-CoV-2. Viral RNA

82 was restricted to cells lining the airways of the lung, in accordance with observations in humans

83 (Fig. 1C) ${ }^{16}$. Examination of the antiviral response to MA-SARS-CoV-2 in the lung found

84 expression of $/ f n / 2$ and ISGs peaked at day 2 p.i. Chemokines $(C x c / 10, C c / 2, C c / 5)$ and endogenous pyrogens (II6, Tnf, I/1b) were upregulated in response to MA-SARS-CoV-2 infection

in the lung (Supplemental Fig. 1B). We next plotted gene expression of representative transcripts

87 against viral RNA and found that $I f i h 1$, Ifn/2, and $/ / 6$ levels positively correlated with MA-SARS-

88 CoV-2 viral burden (Fig. 1D). Thus, MA-SARS-CoV-2 infects the respiratory tract and induces a 89 viral load dependent inflammatory response in C57BI/6 mice.

91 MA-SARS-CoV-2 contains several mutations, including three within the spike protein, at residues

$92 \mathrm{~K} 417 \mathrm{~N}$ and N501Y, which also appear in the SARS-CoV-2 B.1.351 variant ${ }^{17}$. Next, we evaluated

93 if a natural clinical isolate of B.1.351 could establish infection in mice. We intranasally inoculated

94 C57BI/6 mice with $5 \times 10^{5}$ PFU of B.1.351 or MA-SARS-CoV-2 and found that B.1.351 replicated

95 to high viral titers within lungs, as measured by plaque assay and qRT-PCR for RNA-dependent

96 RNA-polymerase (RdRp) RNA and subgenomic (Sg) viral RNA (Fig. 1E). The B.1.351 variant 
97 induced similar levels of cytokines and ISG expression to MA-SARS-CoV-2 in the lung at day 2 98 p.i. (Fig. 1F). Combined these data demonstrate that the variant B.1.351 can infect C57BI/6 mice 99 and replicates similarly to MA-SARS-CoV-2.

100

MA-SARS-CoV-2 infection induces hyper-inflammatory monocytes and dysregulated alveolar macrophages

To investigate cellular innate immunity to SARS-CoV-2, we performed single-cell RNA sequencing (scRNA-Seq) on lung homogenates from day 0 or 4 p.i. with MA-SARS-CoV-2 (4 mice per group). We obtained 9,399 day 0 and 10,982 day 4 p.i. cells and unbiased clustering identified 23 distinct groups comprised of T cells, B cells, DCs, epithelial cells (Epi), neutrophils (Neut), natural killer (NK) cells, alveolar macrophages (AM), and monocytes (mono). We further distinguished between inflammatory (Infl), non-classical (NC), and intermediate (Tr) monocytes (Fig. 2A). MA-SARS-CoV-2 induced a decrease in the frequency of epithelial cells and an increase of inflammatory monocytes and DC frequency in the lung (Fig. 2B).

We next mapped the expression of genes previously associated with COVID-19 progression to

113 cell subsets in our MA-SARS-CoV-2 model (Fig. 2C). We noted low and sporadic expression of

114 Ifnb1 or Ifn/2, but pronounced ISG expression, Isg15 and Irf7, in alveolar macrophage, monocyte,

115 and DC populations. Inflammatory cytokines were expressed primarily in neutrophils (//1b) or

116 monocytes (Cxc/16). Other markers associated with COVID-19, were also localized to neutrophil

117 (S100a8) or monocyte (Mmp14) populations (Fig. 2C) ${ }^{18}$. While the chemokine Cc/2 was 118 expressed primarily by inflammatory monocytes, the cognate receptor, Ccr2, was more 119 widespread with expression on monocytes, DCs, and NK cells (Fig. 2C). Gene set enrichment 120 analysis (GSEA) of inflammatory monocyte populations identified an enrichment of inflammatory, 
121 interferon alpha, and interferon gamma response genes after MA-SARS-CoV-2 infection (Fig. 122 2D). Thus, MA-SARS-CoV-2 results in a pro-inflammatory response driven by neutrophils and 123 inflammatory monocytes.

Alveolar macrophages showed changes in frequency after MA-SARS-CoV-2 infection (Fig. 2B). To further examine this population, we performed heatmap analysis of top differentially expressed genes (DEGs) between mock and MA-SARS-CoV-2 infected samples. MA-SARS-CoV-2 infection upregulated genes involved in antigen presentation (B2m, H2-q7), ISGs (Ifi2712a, Oas1a), and inflammatory cytokines (Cc/9, Cc/6) (Fig. 2E). We performed GSEA using a gene list enriched in alveolar macrophages from COVID-19 patients and found that alveolar macrophages from MASARS-CoV-2 mice had both a hyper-inflammatory and suppressive signature (Fig. 2F) ${ }^{6}$. Together 132 these data demonstrate that alveolar macrophages adopt a dysregulated profile in MA-SARS133 CoV-2 infected mice.

137 Next, we investigated the cellular innate immune response to MA-SARS-CoV-2 at days 0, 2, and

1384 p.i. Mice were intravitally labelled with CD45 conjugated to phycoerythrin (PE) to allow 139 identification of circulating (CD45+ in vivo) and parenchymal (CD45- in vivo) cells in the lung. MA140 SARS-CoV-2 infection initiated a step-wise increase in total circulating and parenchymal CD45+ 141 cell infiltrate in the lung at days 2 and 4 p.i. (Fig. 3A, See Supplemental Fig. 2 for gating strategy).

142 Granulocyte numbers were elevated in circulation at 2 and 4 days p.i. and infiltrated into the lung 143 by day 2 p.i., with a 100-fold increase in parenchymal neutrophils. (Fig. 3B, Supplemental Fig. 1443 A). Circulating macrophage numbers were unchanged by infection, however beginning at day 2 

p.i., parenchymal macrophage numbers decreased as compared to mock-infected mice (Fig. 3C).

146 This downward trend appeared to be due to a sequential loss of alveolar macrophages at day 2 147 and 4 p.i. (Siglec-F+ CD11c+), while interstitial macrophages (CD11c-, SiglecF-, Ly6C-) were 148 unaffected (Fig. 3D). At day 4 p.i., we observed a 100-fold increase in cells that expressed both 149 macrophage markers, CD64 and F4/80, and monocyte markers, Ly6C and CD11b, which we 150 designated 'transitional macrophages' (Fig. 3D). All macrophages upregulated MHC-I in response 151 to MA-SARS-CoV-2, although the effect was more pronounced in transitional macrophages, 152 which also upregulated CD86 (Supplemental Fig. 3B-C). Together these data identify a shift in 153 the lung macrophage composition, with decreased numbers of alveolar macrophages and 154 increased numbers of activated transitional macrophages during MA-SARS-CoV-2 infection.

We next examined the role of dendritic cells during MA-SARS-CoV-2 infection. Plasmacytoid 157 dendritic cell ( $p D C)$ numbers did not change in the circulation or parenchyma at 2-4 days p.i. 158 (Supplemental Fig. 3D). Conventional dendritic cell (cDC) populations remained steady in 159 circulation, but increased 10 -fold in the lung parenchyma at day 4 p.i. that was primarily due to an 160 increase in cDC Type 2 cells (cDC2s) (Fig. 3E, Supplemental Fig. 3E). Lung parenchymal cDCs 161 increased expression of MHC-I and CD86 in response to MA-SARS-CoV-2 at day 4 p.i 162 (Supplemental Fig. 3F). moDCs had slightly increased numbers in circulation and a 10-fold 163 increase in lung parenchymal populations at day 4 p.i. (Fig. 3E). Parenchymal moDCs also 164 upregulated expression of MHC-I and CD86 at day 4 p.i. as compared to uninfected controls 165 (Supplemental Fig. 3G). Thus, cDCs and moDCs undergo expansion and activation in response 166 to MA-SARS-CoV-2 infection in the lung. 

in circulating monocytes and a 20 -fold increase in lung parenchymal monocytes by day 2 p.i. that remained high through day 4 p.i. (Fig. 3F). Ly6C high monocytes drove monocytic infiltration to

171 the lung, as their numbers increased 100-fold at days 2 and 4 p.i., but the numbers of Ly6C low

172 monocytes remained constant (Fig. 3G). All lung parenchymal monocytes showed increased

173 expression of MHC-I at day 4 p.i. (Supplemental Fig. 3H). Ly6C high monocytes had particularly

174 elevated expression of CD86 (2000\% increase) at day 4 p.i. as compared to mock (Supplemental

175 Fig. 3I). Analysis of splenic immunity found that neutrophils had significantly increased numbers

176 at day 4 p.i. (Supplementary Fig. 4A). Dendritic cells showed increased expression of MHC-I at 4

177 days p.i. (Supplementary Fig. 4B). Together these data find that MA-SARS-CoV-2 infection

178 prompts systemic immune activation and a lung parenchymal immune response dominated by

179 the infiltration of activated monocytes and monocyte-derived cells.

\section{Expansion of monocyte-derived cells in the lung parenchyma during MA-SARS-CoV-2}

\section{infection is CCR2-dependent}

183 Classical Ly6C high monocytes migrate to the lung parenchyma in a CCR2-dependent manner 184 and can differentiate into interstitial macrophages or moDCs ${ }^{11,19}$. We next evaluated the contribution of CCR2 signaling in recruiting monocytes to the lung during MA-SARS-CoV-2 infection. Flow cytometry analysis of lungs at day 4 p.i. found similar circulating monocyte numbers, but a 2-fold drop in the number of lung parenchymal infiltrating monocytes in $C c r 2^{-/-}$ mice as compared to WT (Fig. 4A). This decrease appeared to be driven by a 4-fold drop in Ly6C high and Ly6C intermediate monocytes in the lungs of $\mathrm{Ccr}^{-/-}$mice (Fig. 4B). Circulating moDC numbers were unaltered by the absence of CCR2, but lung parenchymal moDCs dropped 10-fold 191 in Ccr2 $^{-/-}$mice at day 4 p.i. (Fig. 4C). cDC numbers in circulation were similar between WT and $192 \mathrm{Ccr}^{-/}$mice at day 4 p.i., but lung parenchymal cDC numbers at day 4 p.i. were 5 -fold lower than 
193 WT (Fig. 4D). This was due to a specific loss in cDC2s (Fig. 4D). All monocyte subsets showed

194 decreased expression of CD86 (2-fold decrease) at day 4 p.i. in Ccr2 ${ }^{-/-}$mice, while MHC-I levels

195 were decreased only in the Ly6C intermediate subset (Fig. 4E). Expression of antigen

196 presentation markers on moDCs was unchanged by CCR2 (Supplemental Fig. 5A). Lung-

197 infiltrating $\mathrm{CDC} 2 \mathrm{~s}$ from $\mathrm{Ccr} 2^{-/-}$mice had lower expression of MHC-I, but not CD86 as compared to

198 WT cells at 4 days p.i. (Supplemental Fig. 5B). Together these data find that CCR2 signaling

199 promotes the infiltration of activated Ly6C high and intermediate monocytes, moDCs, and cDC2s

200 into the lung parenchyma during MA-SARS-CoV-2 infection.

201

202 We investigated if CCR2 low/negative innate immune cells (Fig. 3C) were impacted by secondary

203 effects of CCR2. The total number of macrophages in circulation or in the lung parenchyma was

204 not affected by CCR2 at 4 p.i. with MA-SARS-CoV-2 (Fig. 4F). Alveolar and interstitial

205 macrophages were not impacted by CCR2 signaling. However, Ccr2 ${ }^{-/}$mice had a 10-fold drop in

206 transitional macrophage numbers as compared to WT mice (Fig. 4G). Expression of CD86 and

207 MHC-I was also decreased on transitional macrophages from Ccr2 ${ }^{-/}$mice at day 4 p.i. Despite

208 similar numbers in WT and $\mathrm{Ccr}^{-/-}$mice, interstitial macrophages failed to upregulate expression

209 of both MHC-I and CD86 in $\mathrm{Ccr}^{-/-}$mice at day 4 p.i. Expression of CD86 MFI on alveolar

210 macrophages from $\mathrm{CCr}^{-/-}$mice was modestly decreased compared to WT (Fig. 4H). Thus,

211 activation of macrophages and expansion of transitional macrophages is CCR2 dependent during

212 MA-SARS-CoV-2 infection.

214 Lung parenchymal granulocyte numbers were not significantly altered between WT and Ccr2 ${ }^{-/-}$

215 mice at day 4 p.i. (Supplemental Fig. 5C). However, there was a modest increase in the number

216 of granulocytes in circulation and the spleen from $\mathrm{Ccr}^{-/-}$mice as compared to WT mice at day 4 
217 p.i. (Supplemental Fig. 5C-D). Total splenic macrophage numbers were also higher in the 218 absence of CCR2 at day 4 p.i. (Supplemental Fig. 5D). Monocyte, moDC, and pDC populations 219 in the spleen were unaffected by CCR2, however numbers of cDCs in the spleen were increased 220 in $\mathrm{Ccr}^{-/}$mice at day 4 p.i. as compared to WT (Supplemental Fig. 5D). These data identify a role 221 for CCR2 in promoting the infiltration of activated, Ly6C high monocytes and monocyte derived 222 cells to the lung during MA-SARS-CoV-2 infection.

225 To determine if CCR2 was protective against MA-SARS-CoV-2, we infected WT or Ccr2 ${ }^{-/}$mice 226 with MA-SARS-CoV-2 and assessed viral burden at day 4 p.i. Ccr2-- mice had 10-fold higher viral 227 burden in lung tissue as measured by plaque assay or qRT-PCR (Fig. 5A-B). At day 4 p.i., Ifn/2, 228 cytokines (I/6), and chemokines (Cxc/10, Cc/2) were elevated in $\mathrm{Ccr}{ }^{-/-}$as compared to WT lungs 229 (Fig. 5C). In situ hybridization found that $\mathrm{Ccr}^{-/}$lungs had more robust detection of viral RNA than 230 WT mice. Additionally, while viral RNA was localized to cells lining the airway spaces in WT mice, $231 \mathrm{Ccr} 2^{-/-}$lungs had infiltration of viral RNA further into the interstitial and parenchymal spaces (Fig.

232 5D). Together, these data find that CCR2 mediated signaling and immune cell recruitment restrict 233 inflammation, viral burden, and viral dissemination in the lung during MA-SARS-CoV-2 infection.

\section{Discussion}

236 SARS-CoV-2 infection in humans has identified a robust and reproducible correlation between 237 inflammatory cytokine levels and disease severity during COVID-196. In accordance, we found 238 that SARS-CoV-2 infected mice had a pro-inflammatory cytokine profile in the lung containing 239 pyrogens (I/6, Tnf), chemoattractants for monocytes (Cc/2) and T cells (Cxc/10), ISGs (IIf7 and 240 Isg15), alarmins (S100a8), and matrix metalloproteinases $(M m p 14)^{6,20-23}$. Interestingly, 
241 inflammatory gene expression in the lung was MA-SARS-CoV-2 viral load dependent, as most

242 cytokine transcripts surveyed by qPCR positively correlated with viral RNA. Similar phenomenon

243 have been noted in human subjects, with one study describing an association between SARS-

244 CoV-2 viral burden, IL-6 levels, and increased risk of death ${ }^{24}$. Similar to studies of post-mortem

245 lung tissue or BALs from patients suffering from COVID-19, we observed a significant increase in

246 the numbers of S100a8+ granulocytes in the lung parenchyma ${ }^{4,6-8}$. S100a8/9 and TLR4 signaling

247 mediate the emergence of a dysregulated neutrophil population that promotes SARS-CoV-2

248 disease ${ }^{18}$. Neutrophil and inflammatory cytokine levels were higher in $\mathrm{Ccr}^{-/-}$mice, likely driven

249 by increased viral burden in $\mathrm{Ccr}^{-/-}$lungs. Thus, MA-SARS-CoV-2 viral burden is likely directly

250 driving cytokine expression and neutrophilic infiltration into the lung.

252 CCR2 expression is a defining feature of inflammatory Ly6C high blood monocytes ${ }^{9}$. In various

253 models of respiratory disease including influenza, Mycobacterium tuberculosis, and allergic

254 inflammation, deletion of Ccr2 negates the ability of monocytes to enter the lung

255 parenchyma ${ }^{11,25,26}$. Similarly, we found that CCR2 had no effect on circulating monocyte numbers

256 but specifically promoted infiltration of activated monocytes into the lung parenchyma during

257 SARS-CoV-2. Monocyte-derived interstitial macrophages and moDCs also depended on CCR2

258 for expansion in the lung parenchyma ${ }^{27}$. In contrast, alveolar macrophages, which are self-

259 renewing and primarily fetal derived, were unaffected by the absence of CCR2 $2^{28-30}$. Previous

260 studies in a model of sterile lung inflammation demonstrated that DC precursors required CCR2

261 for entry to the lung, and accordingly in MA-SARS-CoV-2 infection, cDC2 lung parenchymal

262 populations were also decreased in the absence of CCR2 ${ }^{31}$. These data suggest that CCR2 plays

263 an essential and specific role in promoting the recruitment and differentiation of monocytes into

264 transitional macrophage and moDC populations during MA-SARS-CoV-2 infection. 
266 In contrast to other respiratory infections such as influenza, in which CCR2 promotes mortality

267 and inflammation, our study found that CCR2 restricted viral burden and weight loss during MA-

268 SARS-CoV-2 infection ${ }^{11}$. CCR2 promoted the infiltration of monocyte-derived cells into the lung 269 parenchyma, thus suggesting an essential role for Ly6C high monocytes, moDCs, and transitional 270 macrophages in controlling SARS-CoV-2 infection. Macrophages have been previously shown to 271 control viruses through the secretion of iNOS and the phagocytosis of viral particles, while moDCs 272 play a key role in Type I IFN release and priming the T cell response ${ }^{13,32}$. Further studies are 273 needed to delineate the precise mechanisms these cell subsets use to control SARS-CoV-2. 274 Current evidence in the field of SARS-CoV-2 research suggests that a large portion of the 275 pathology of COVID-19 is due to a monocyte-driven cytokine storm ${ }^{33}$. Here we identify data that 276 suggests monocyte-derived cells in the lung are crucial for limiting viral burden and cytokine 277 production during the early stages of MA-SARS-CoV-2 infection. Most studies to date of the 278 immune response in humans have focused on late-timepoints of infection, therefore monocytes 279 could be protective early and pathological later during infection resolution. The consideration of 280 the role of monocyte-derived cells in restricting SARS-CoV-2 infection in the lung and priming 281 adaptive immune responses will be essential for the design of future therapies and vaccines. 


\section{Figure 1}

A.

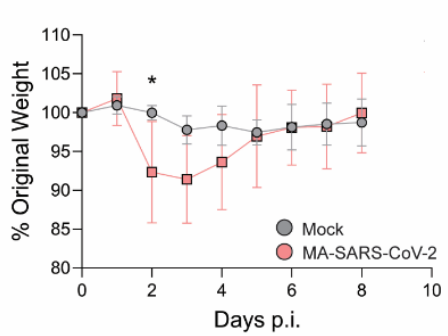

c.

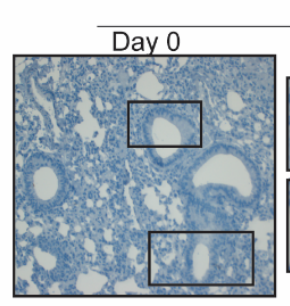

MA-SARS-CoV-2

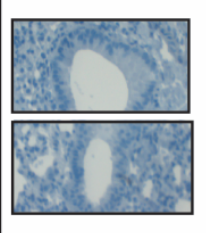

F.

E.

Day 2

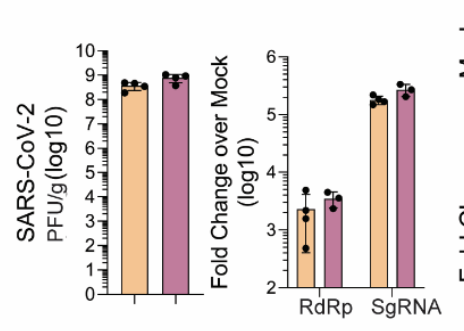

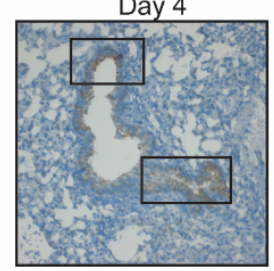

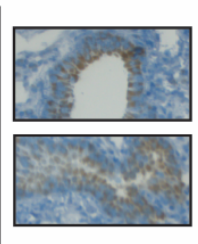

B.

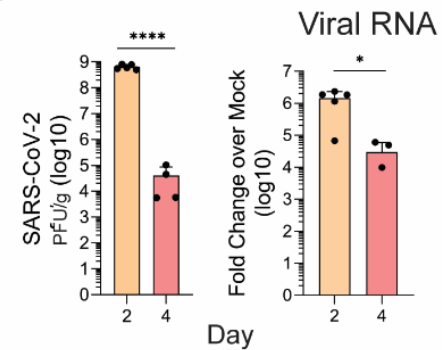

Day 2

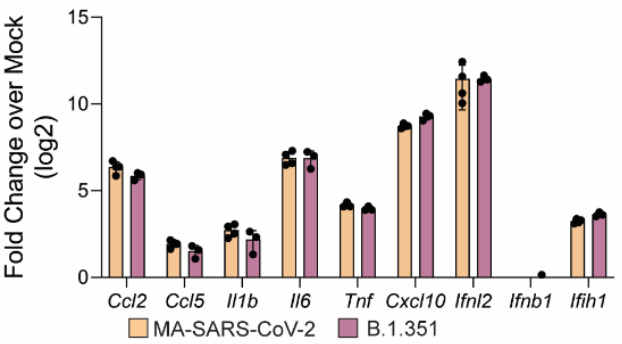

D.
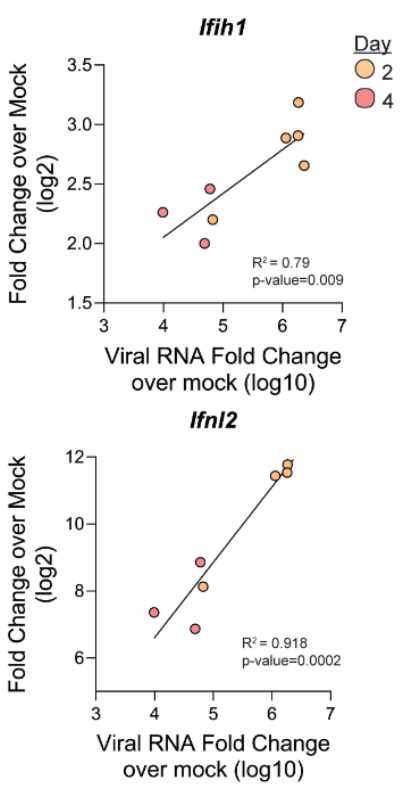

II6

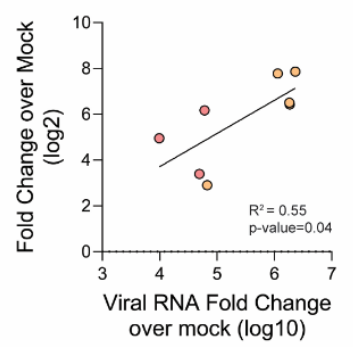

Figure 1. MA-SARS-CoV-2 and B.1.351 replicate in the respiratory tract. C57BI/6J mice were

287 infected intranasally with $5 \times 10^{5}$ PFU of MA-SARS-CoV-2 or an equal volume of PBS for mock measured by plaque assay (left) or qRT-PCR (right). $\mathrm{C}_{\mathrm{T}}$ values are represented as relative fold change over mock $\left(\log _{10}\right)$. C) In situ hybridization was performed using a probe for MA-SARSCoV-2 spike protein RNA. Representative images of lung slices from mock or day 4 p.i. D) Fold- 
2942 viral RNA for each sample, and linear regression was used to determine correlation. E) 295 Quantification of viral titers from lung tissue by plaque assay at day 2 p.i. from mice infected with 296 MA-SARS-CoV-2 or human variant B.1.351 (5 x 105 PFU/mouse). On the right, quantification of 297 RNA-dependent RNA-polymerase (RdRp) or subgenomic RNA (SgRNA) fold change over mock. 298 F) Gene expression measured via qRT-PCR for the indicated gene from lungs infected with MA299 SARS-CoV-2 or B.1.351 at 2 days p.i. Results are representative of 2 independent experiments 300 with 5 mice per group. Statistical significance was determined using unpaired student's t-tests or 301 linear regression. ${ }^{*} p<0.05,{ }^{* * * *} p<0.0001$ 


\section{Figure 2}

A

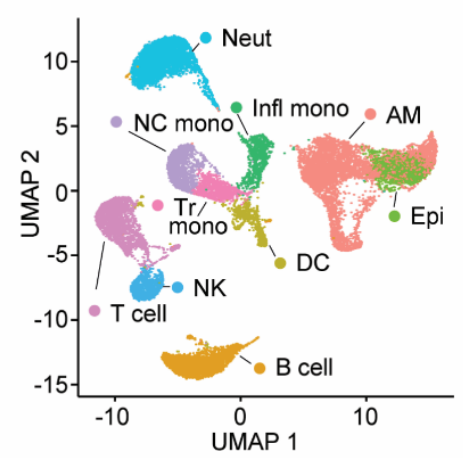

B

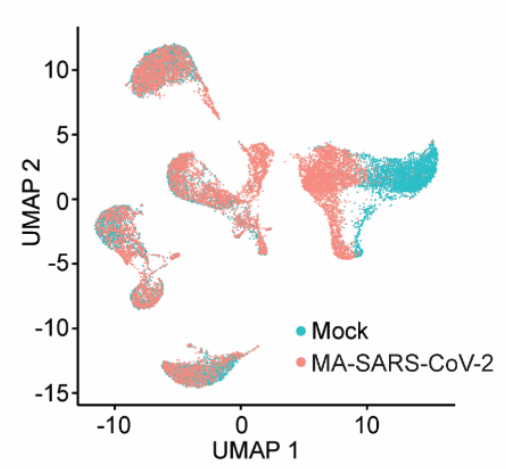

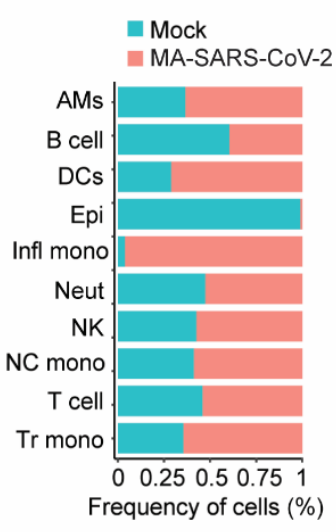

C
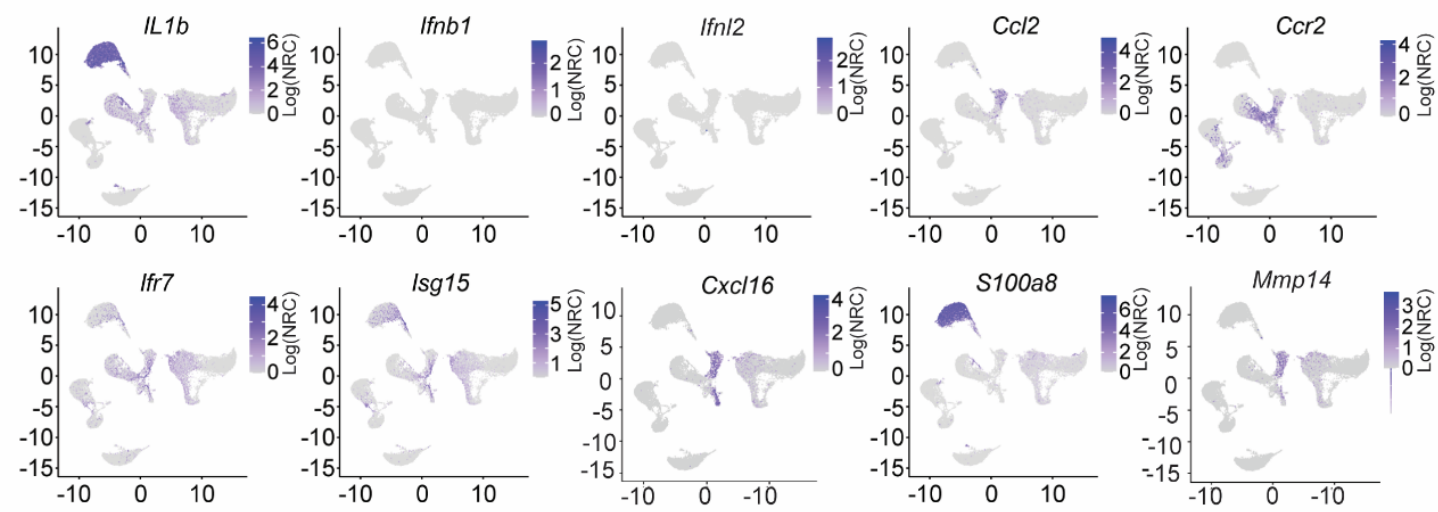

E

D
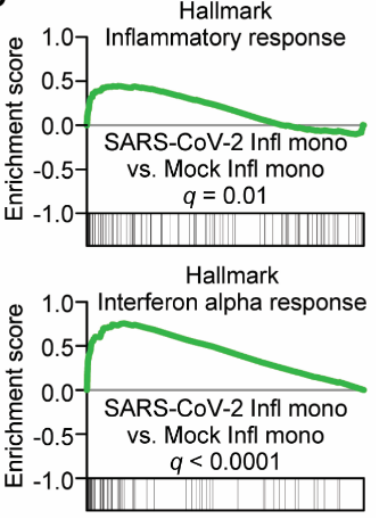

Hallmark

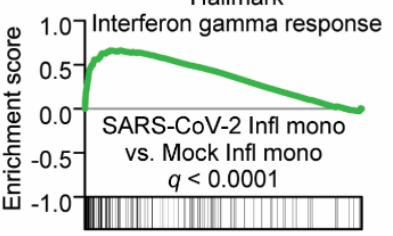

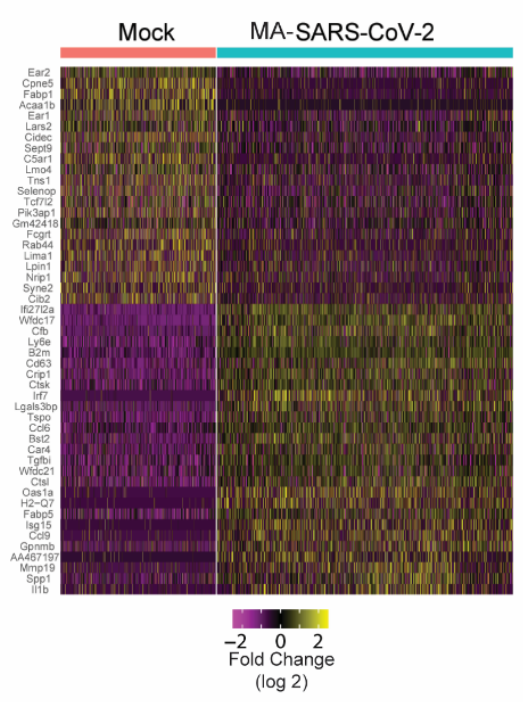
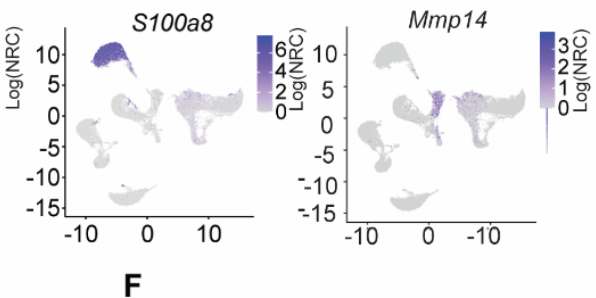

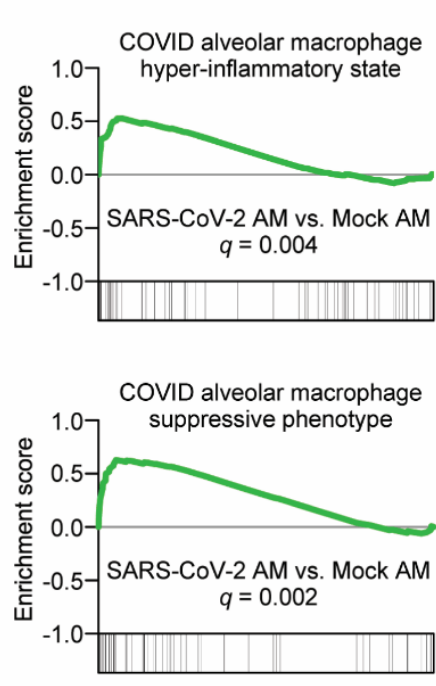


305 Figure 2. MA-SARS-CoV-2 induces hyper-inflammatory monocytes and macrophages in

306 the lung. C57BI/6 mice were infected with MA-SARS-CoV-2 and lungs were harvested at day 0

307 and 4 p.i., processed to a single cell suspension, captured in droplets on a 10X Chromium

308 controller, and analyzed via scRNA-Seq ( $n=4$ per group). A) UMAP plot illustrating the different

309 cellular subsets identified in the lung. B) UMAP distribution of cells from mock or MA-SARS-CoV-

3102 infected mice. On the right, the frequency of mock vs infected cells that make up each subset

311 defined by UMAP analysis. C) Feature plots displaying the average gene expression of the

312 indicated gene from mock and infected lungs. D) GSEA of inflammatory monocytes using the

313 hallmarks database from MSigDB for the indicated gene set. E) Heatmap analysis of top scoring

314 DEGs in alveolar macrophages from mock or SARS-CoV-2 infected lungs. F) GSEA plots of the

315 indicated gene set from Liao et al. in alveolar macrophages from mock and infected lungs. 


\section{Figure 3}

A.

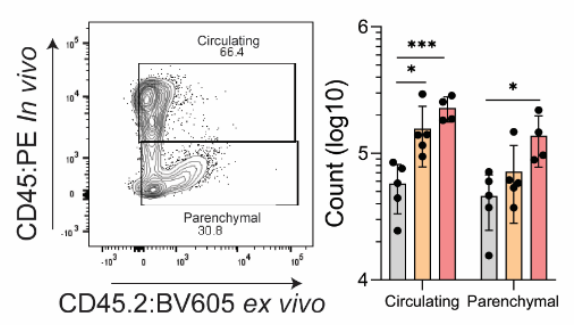

B.

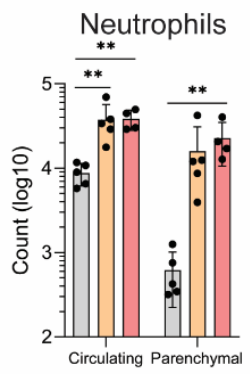

C. Macrophages

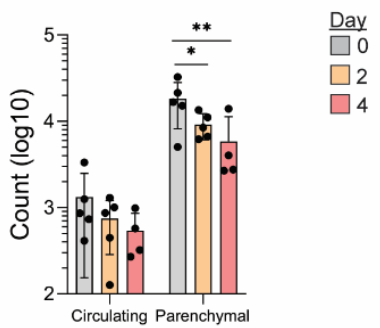

D.

Parenchymal Macrophage Subsets

E.
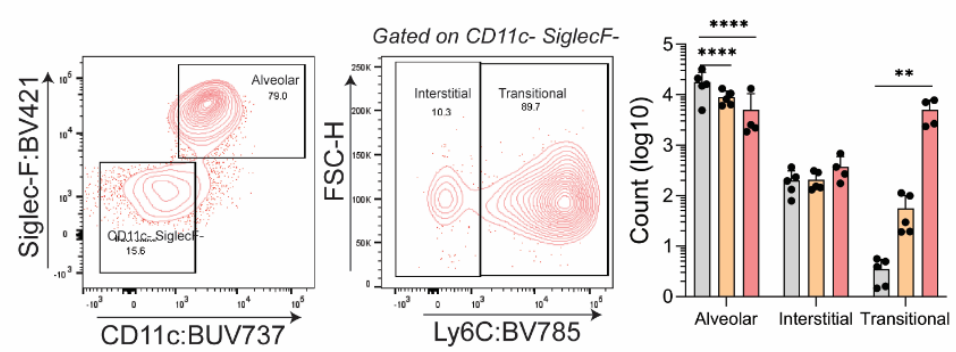

cDCs

moDCs
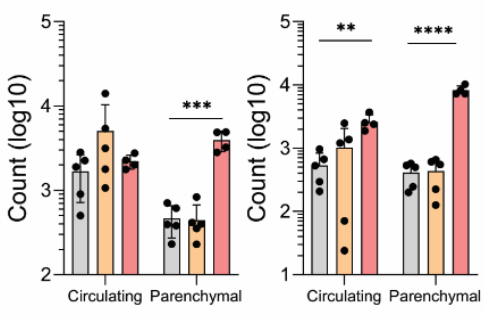

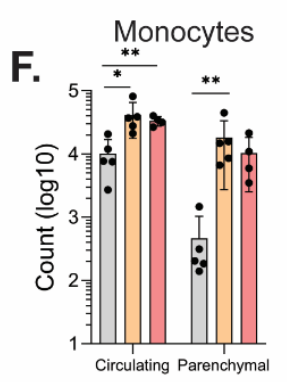

G.

Parenchymal Monocyte Subsets
Day 0
Day 4
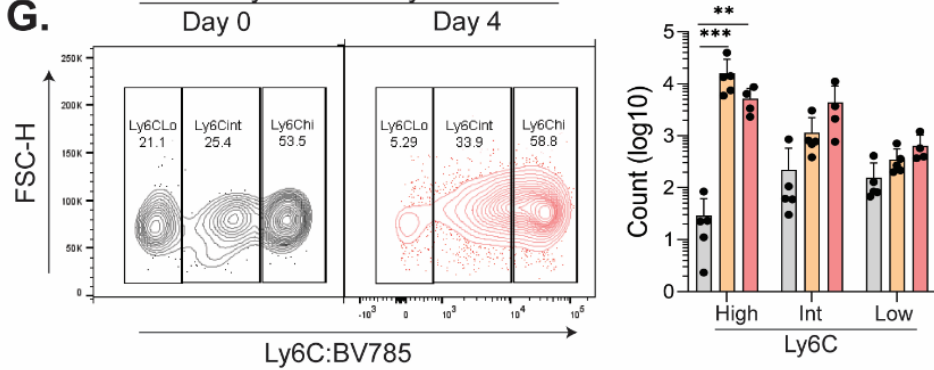

Figure 3. Monocytes and monocyte-derived cells rapidly infiltrate the lung parenchyma in response to MA-SARS-CoV-2 infection. C57BI/6 mice were infected with MA-SARS-CoV-2 and lung tissue was harvested at 0,2 , and 4 days p.i. and analyzed via flow cytometry. A) 5 minutes prior to euthanization, mice were intravitally labelled with CD45:PE. Representative gating of in

322 vivo labelled CD45+ cells used to identify lung circulating (CD45+ in vivo) or lung parenchymal

323 (CD45- in vivo) cells. The total number of CD45+ ex vivo cells is quantified on the right. B) Counts of neutrophils (Lineage-, CD11b+, Ly6G+) over the course of infection. C) Counts of macrophages

325 at day 0, 2, and 4 p.i. (Lineage-, Ly6G-, CD64+, F4/80+). D) Representative flow gating for 
327 , Ly6C+) macrophages from day 4 p.i. Quantified on the right are the counts of each population.

328 E) Quantification of cDCs (lineage-, Ly6G-, CD64-, MHCII+, CD11C+, CD26+) or moDCs (lineage329 , Ly6G-, MHC-II+, CD11b+, CD11c+) counts at the indicated timepoint. F) Total monocyte 330 (lineage-, Ly6G-, MHC-II-, CD11C-, CD64 low) counts for circulating or lung parenchymal cells at 331 day 0, 2, and 4 p.i. G) Representative gating strategy to identify different monocyte subsets (Ly6C 332 High, Ly6C Intermediate (Int), Ly6C low), and the quantification on the right. Results are 333 representative of two independent experiments with 5 mice per group. Statistical significance 334 was determined using unpaired one or two-way ANOVA. ${ }^{*} p<0.05,{ }^{* *} p<0.01,{ }^{* * *} p<0.001$, $335{ }^{* * * *} p<0.0001$. 


\section{Figure 4}

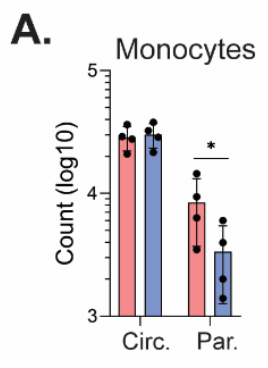

C.

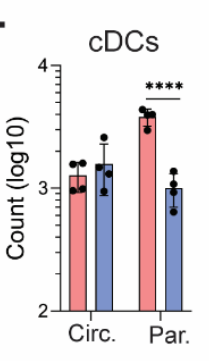

B.

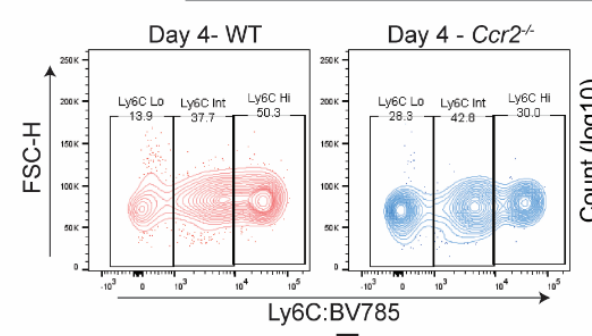

E.
Parenchymal Monocyte Subsets

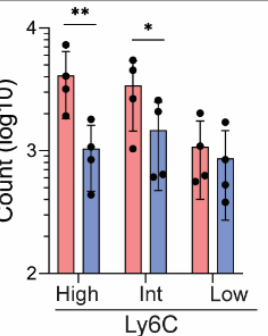

Parenchymal cDCs
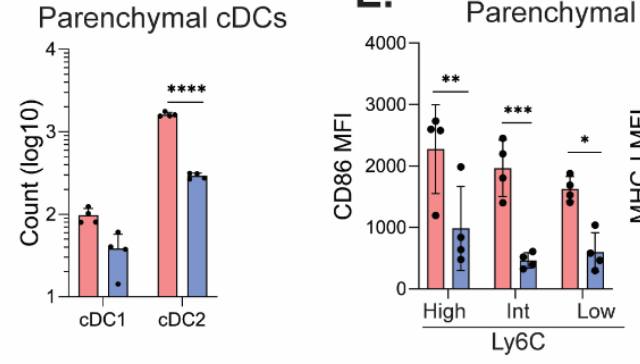

G.

Parenchymal Macrophage Subsets
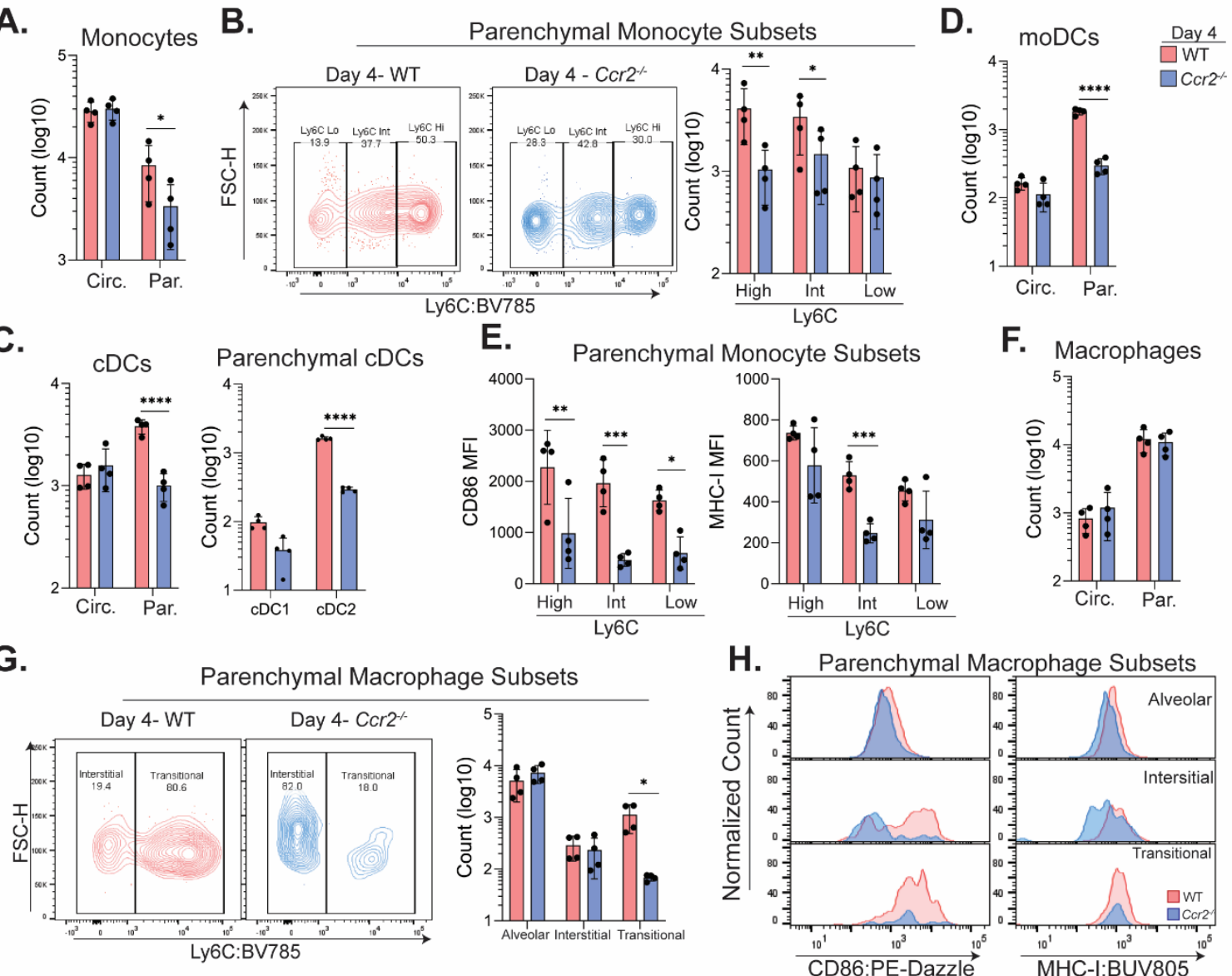

F. Macrophages

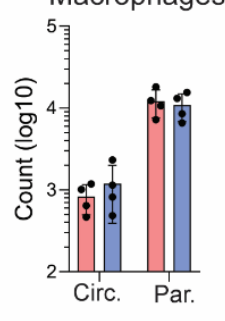

H. Parenchymal Macrophage Subsets

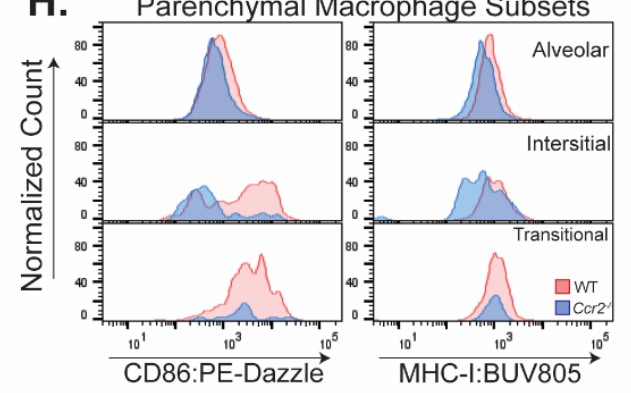

Figure 4. Expansion of monocyte-derived cells in the lung during MA-SARS-Cov-2 is CCR2 dependent. C57BI//6 and $\mathrm{Ccr}^{-/-}$mice were infected with MA-SARS-CoV-2 and lung tissue was harvested at 0 and 4 days p.i. and analyzed via flow cytometry. Circulating (Circ.) vs parenchymal

341 (Par.) cells were distinguished as described in Fig. 2. A) Number of total monocytes in the lung circulation or parenchyma B) Representative gating identifying Ly6C High, Intermediate (Int), or Low monocytes from WT and $\mathrm{Ccr}^{-/}$lung parenchyma. Counts for each subset are quantified to

344 the right. C) Quantification of moDCs at day 4 p.i. D) Total numbers of cDCs and parenchymal cDC subsets (right) E) MFI for CD86 (left) and MHC-I (right) expression on monocyte subsets from the lung parenchyma. F) Quantification of the total macrophage numbers at day 4 p.i. G)

347 Representative flow plots illustrating interstitial or transitional macrophage populations from WT 
348 and $\mathrm{Ccr}^{-/-}$lung infiltrating cells. Counts of macrophage subsets are quantified on the right. $\mathrm{H}$ )

349 Representative histograms of the MFI for CD86 (left) and MHC-I (right) for each macrophage

350 subset 4 days p.i. from WT or $\mathrm{Ccr}^{-/-}$lung infiltrating cells. Results are representative of two

351 independent experiments with 5 mice per group. Statistical significance was determined using

352 unpaired one or two-way ANOVA. ${ }^{*} p<0.05,{ }^{* *} p<0.01,{ }^{* * *} p<0.001,{ }^{* * *} p<0.0001$. 


\section{Figure 5}

A.

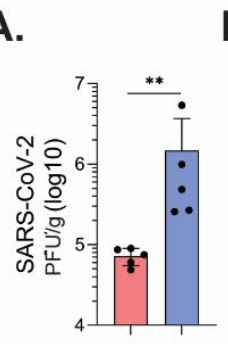

B.

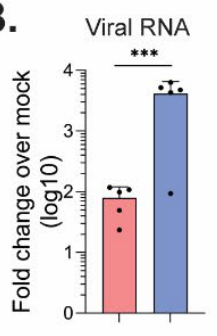

C.

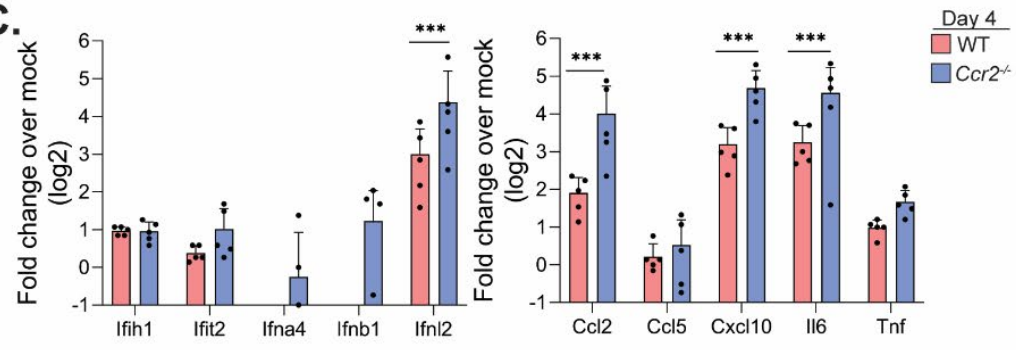

D.

Mock

MA-SARS-CoV-2
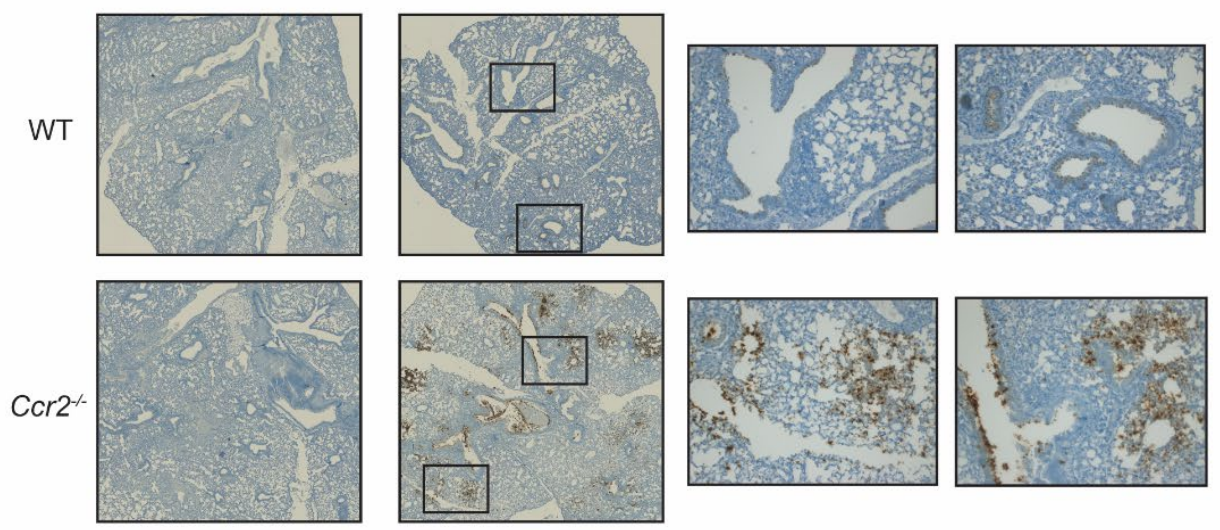

Figure 5. CCR2 restricts MA-SARS-CoV-2 viral burden and inflammatory cytokines in the

lung. C57BI/6 or $\mathrm{Ccr}^{-/-}$mice were infected with MA-SARS-CoV-2 and lung tissue was collected at day 4 p.i. A) Infectious virus at day 4 p.i. as quantified via plaque assay. B) qRT-PCR for the SARS-CoV-2 RdRp. C) qRT-PCR was performed to probe for the indicated IFN signaling (left) or inflammatory (right) transcripts. D) Representative images of in situ hybridization to visualize MASARS-CoV-2 RNA in lung tissue slices from 0 and 4 days p.i. in both WT and $C \mathrm{cr} 2^{-/}$mice. Results are representative of two independent experiments with 5 mice per group. Statistical significance 


\section{Supplemental Figure 1.}

A

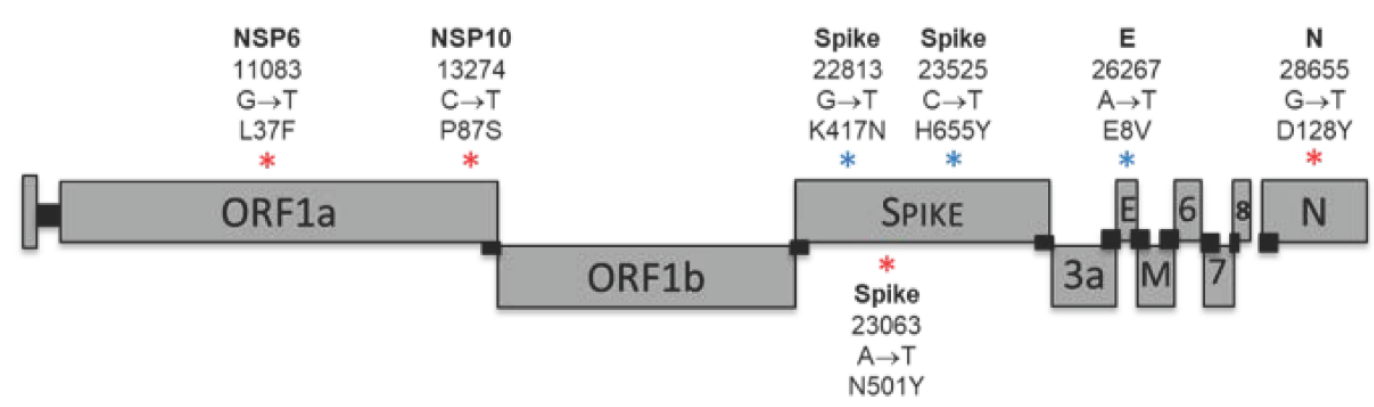

B

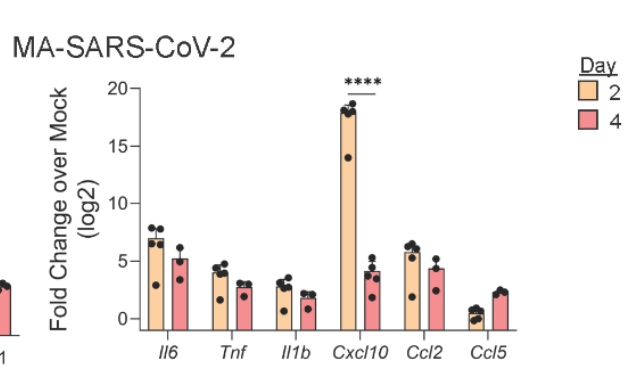

Supplemental Figure 1. MA-SARS-CoV-2 induces an inflammatory response in the lung. A)

Schematic of MA-SARS-CoV-2. Mutations with a red asterisk were engineered into icSARS-CoV-

3702 followed by passaging the virus in Balb/c mice. Following 20 passages, the virus was sequenced

371 and mutations with a blue asterisk were acquired in addition to the engineered mutations during

372 the passage of MA-SARS-CoV-2. B) Mice were treated with PBS or infected with MA-SARS-CoV-

3732 via the intranasal route and lung tissue was harvested from 0,2 , and 4 days p.i. Fold change

374 over mock for the indicated genes at day 2 or 4 p.i. with MA-SARS-CoV-2. Results are

375 representative of 2 independent experiments. Statistical significance was determined using 376 unpaired two-way ANOVA. ${ }^{* * *} p<0.001,{ }^{* * * *} p<0.0001$. 


\section{Supplemental Figure 2.}
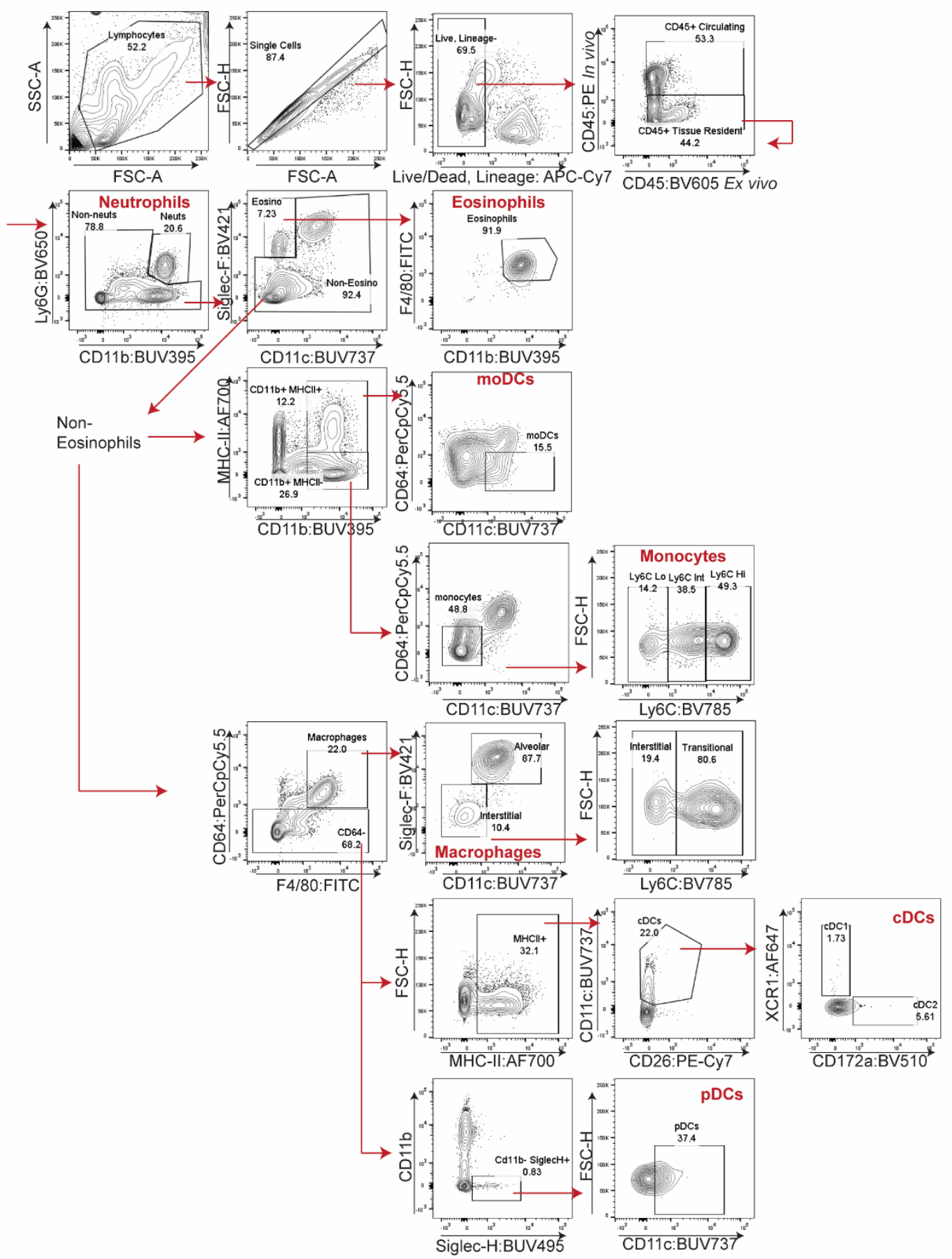


\section{Supplemental Figure 2. Gating strategy for innate immune populations in the mouse lung.}

380 C57BI/6 mice were infected with MA-SARS-CoV-2 and lung tissue was harvested at 0, 2 and 4 381 days p.i. 5 minutes prior to euthanization, mice were intravitally labelled with CD45:PE (injected 382 via the retro-orbital route). Lungs were processed to a single-cell suspension and analyzed via 383 flow cytometry. Total cell populations were gated on (FSC-SSC), then singlets, then on Live

384 (Ghost-Dye-) and Lineage- (CD19, CD3, NK1.1) populations. Lung infiltrating cells were identified 385 as CD45 ex vivo+ and CD45 in vivo-. All following gates were made for both circulating and lung 386 parenchymal populations. Neutrophils were identified as Ly6G+, CD11b+, CD11c-, and removed 387 from downstream analysis. Eosinophils were identified as Siglec-F+, CD11c-, F4/80+, CD11b+, 388 and removed from downstream analysis. Monocytes were identified as CD11b+, MHC-II-, CD11c389 , CD64 ${ }^{\text {low }}$. moDCs were identified as CD11b+, MHC-II+, CD64 low, CD11c+. Macrophages were 390 identified as CD64+, F4/80+, and distinguished into subsets based on Siglec-F, CD11c, and Ly6C 391 expression. cDCs were identified as CD64-, MHC-II+, CD11c+, CD26+. cDC1s and cDC2s were 392 distinguished by XCR1 and CD172a staining. pDCs were identified as CD11b-, Siglec-H+, and 393 CD11C+. Subsequent gating is indicated by red arrows, and all final populations used for analyses 394 are labelled in red. Flow plots are representative of lung populations from day 4 post MA-SARS395 CoV-2 infection. 


\section{Supplemental Figure 3.}

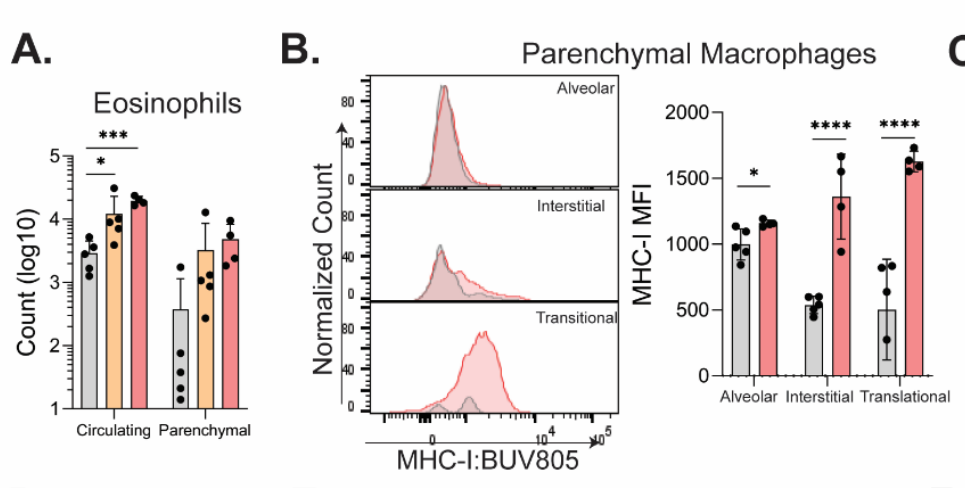

D. $\mathrm{pDCs}$

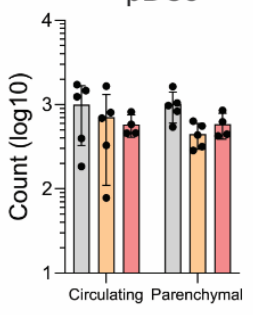

E.

Parenchymal cDC Subsets

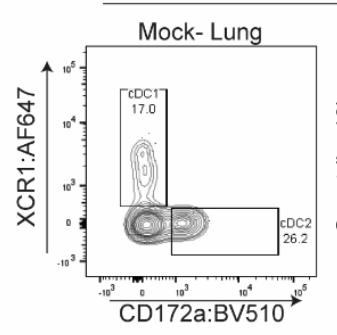

F.
C.

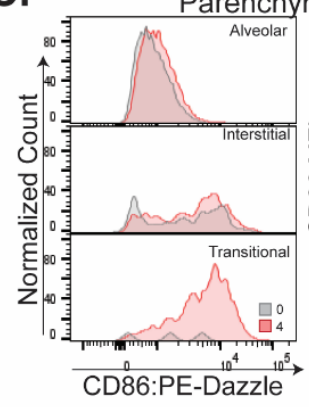

Parenchymal Macrophages $\quad \frac{\text { Day }}{\square 0}$
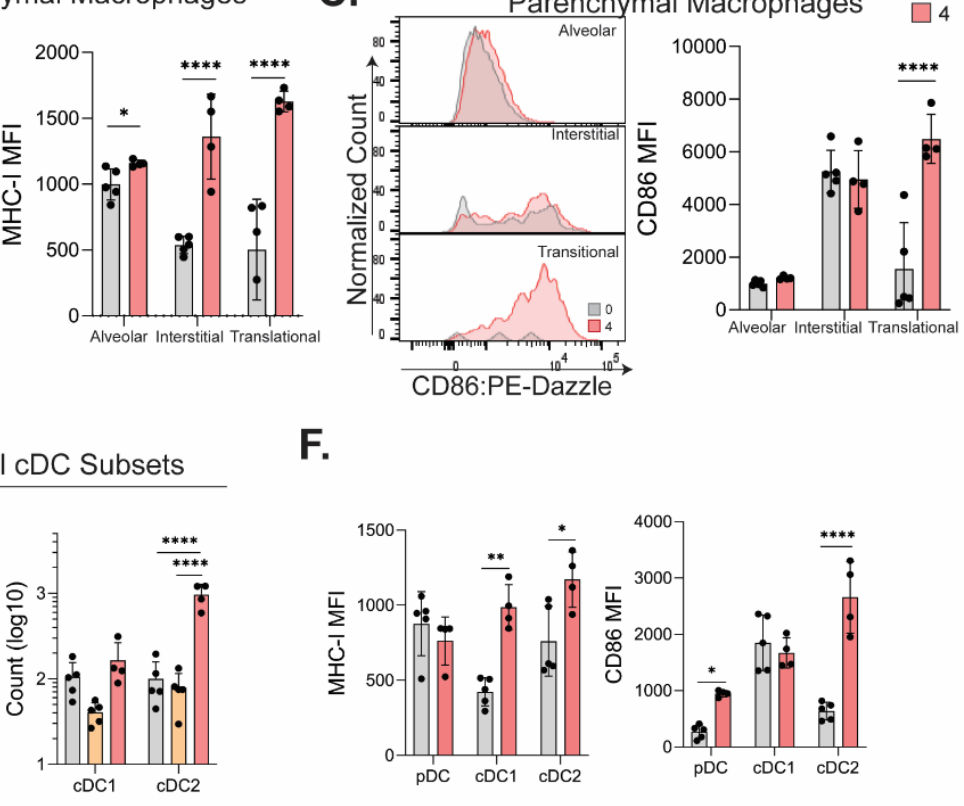
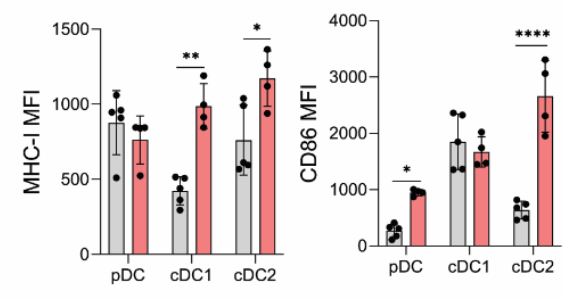

G.
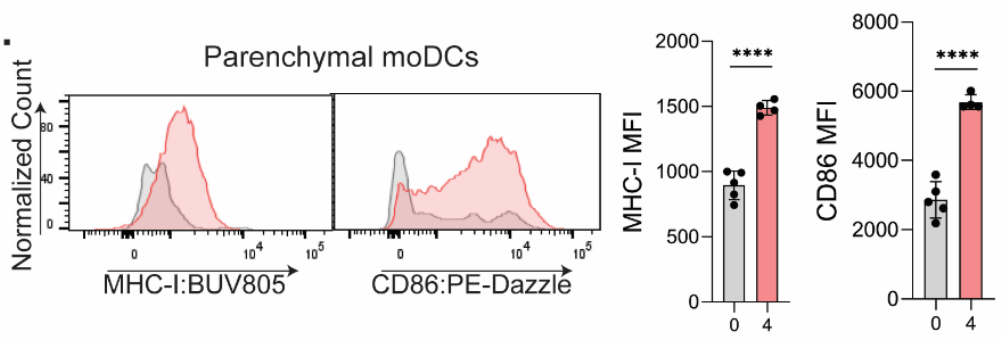

H. Parenchymal Monocytes
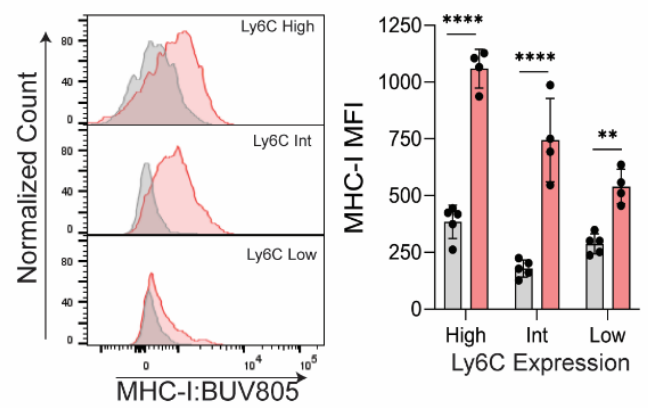

I.
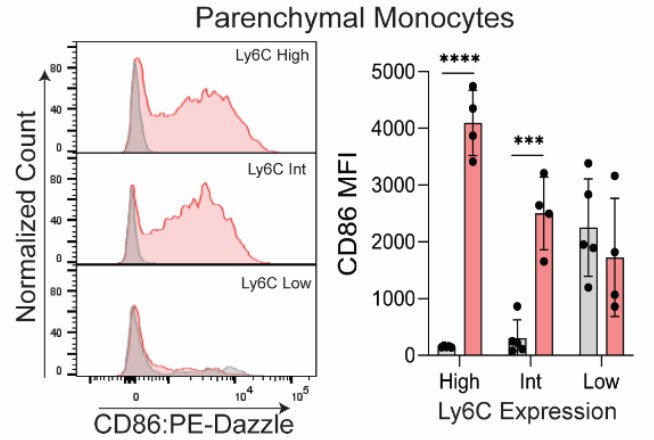

Day

品 
to MA-SARS-CoV-2. C57BI/6 mice were infected intranasally with MA-SARS-CoV-2, harvested

400 at day 0,2 , or 4 p.i., and lung parenchymal vs circulating cells were identified as described in Fig.

401 2. A) Quantification of eosinophils (Lineage-, Ly6G-, CD11c-, SiglecF+, F4/80+, CD11b+).

402 Representative histograms of B) MHC-I or C) CD86 expression on macrophage subsets.

403 Quantified on the right are mean fluorescent intensities for day 0 and 4 p.i. D) Quantification of

404 the number of pDCs (Lineage-, Ly6G-, CD64-, CD11b-, SiglecH+, CD11c+). E) Representative

405 flow plot of cDC subset gating strategy and quantification of cDC1s (XCR1+, CD172a-) or cDC2s

406 (XCR1-, CD172a+) on the right. F) Quantification of the MFI for MHC-I and CD86 on pDC and

407 cDC subsets in the parenchyma at day 0 and 4 p.i. G) Representative histograms of MHC-I and

408 CD86 expression on parenchymal moDCs at day 0 and 4 p.i., with the quantification on the right.

409 Representative histograms for H) MHC-I or I) CD86 expression on lung parenchymal monocyte

410 subsets and the corresponding quantification on the right. Results are representative of two

411 independent experiments. Statistical significance was determined using unpaired one or two-way

412 ANOVA. ${ }^{*} p<0.05,{ }^{* *} p<0.01,{ }^{* * *} p<0.001,{ }^{* * *} p<0.0001$ 


\section{Supplemental Figure 4.}

A.

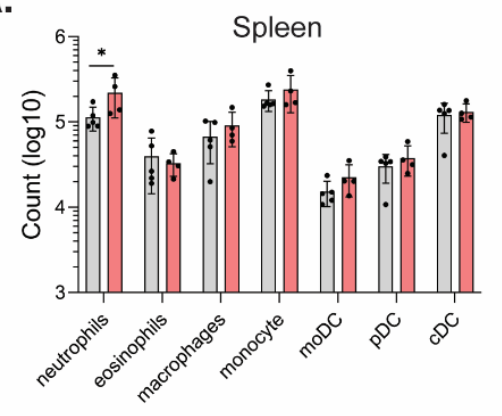

B.

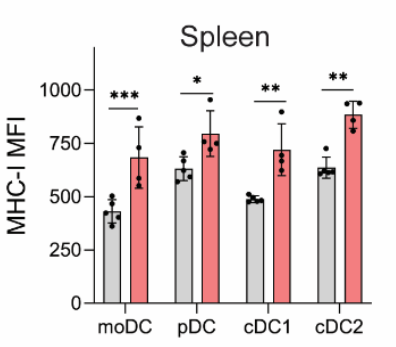

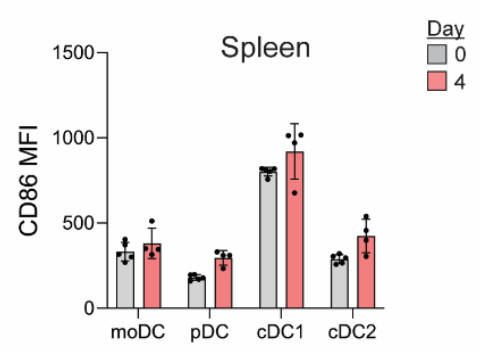

415 Supplemental Figure 4. MA-SARS-CoV-2 infection induces systemic activation of dendritic

416 cells. WT mice were infected with MA-SARS-CoV-2, and spleens were harvested at day 0 or 4

417 p.i. A) Quantification of the indicated cell populations in the spleen at day 0 or 4 p.i. D) MFls for 418 MHC-I (left) and CD86 (right) expressed on splenic dendritic cell populations. Results are 419 representative of two independent experiments. Statistical significance was determined using 420 unpaired one or two-way ANOVA. ${ }^{*}=p<0.05,{ }^{* *}=p<0.01,{ }^{* * *}=p<0.001$. 


\section{Supplemental Figure 5.}

A.

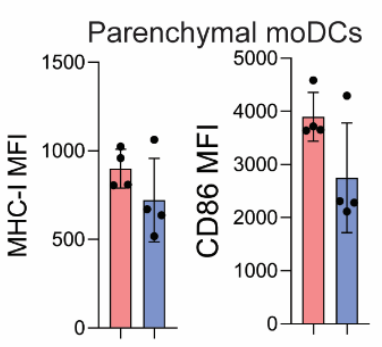

C.

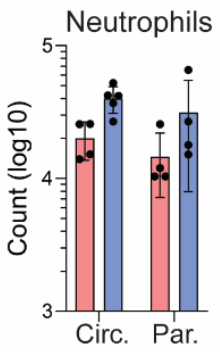

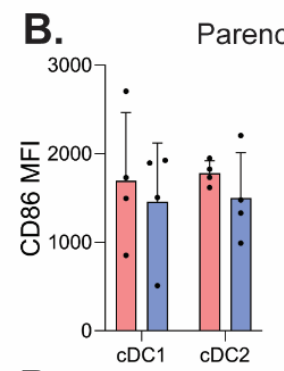

D.

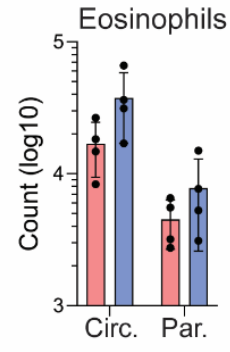

Spleen

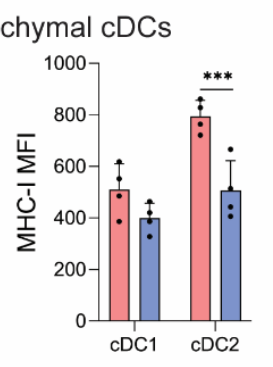

$\frac{\text { Day } 4}{\square W T}$
$\square$ Ccr ${ }^{-}$

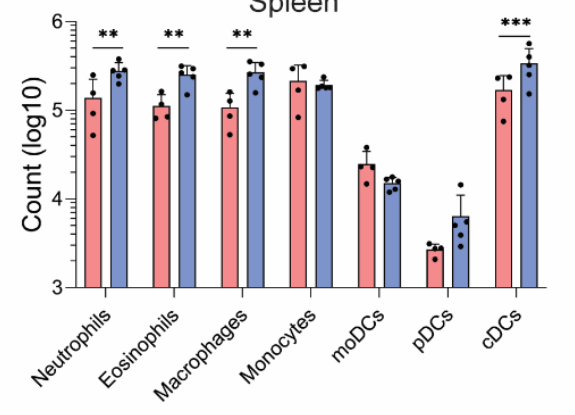

423 Supplementary Figure 5. CCR2 limits systemic expansion of innate immune cells. WT and

$424 \mathrm{Ccr} 2^{-/}$mice were infected with MA-SARS-CoV-2, and lung tissue and spleens were harvested at 425 day 0 and 4 p.i. Lung-infiltrating cells were identified by intravital labelling and analyzed via flow 426 cytometry. A) MFIs for MHC-I and CD86 expression on lung-infiltrating moDCs or B) CDC subsets.

427 C) Quantification of the indicated granulocyte population in the lung. D) Quantification of the 428 indicated innate immune cell population in the spleen. Results are representative of two 429 independent experiments. Statistical significance was determined using unpaired student's t-test or two-way ANOVA. ${ }^{*}=p<0.05,{ }^{* *}=p<0.01,{ }^{* * *}=p<0.001$. 


\section{METHODS}

433 Viruses and cells. VeroE6 cells were obtained from ATCC (clone E6, ATCC, \#CRL-1586) and

434 cultured in complete DMEM medium consisting of 1x DMEM (VWR, \#45000-304), 10\% FBS,

435 25mM HEPES Buffer (Corning Cellgro), 2mM L-glutamine, 1mM sodium pyruvate, 1x Non-

436 essential Amino Acids, and 1x antibiotics. VeroE6-TMPRSS2-hACE2 cells were kindly provided

437 by Dr. Barney Graham (Vaccine Research Center, NIH, Bethesda, MD). The generation of the

438 MA-SARS-CoV-2 virus will be described in a future publication. In brief, MA-SARS-CoV-2 virus

439 was generated by engineering four coding mutations (NSP6 L37F, NSP10 P87S, S N501Y, and

$440 \mathrm{~N} \mathrm{D128Y)}$ into the backbone of icSARS-CoV-2 (WA/1 backbone). This virus was then passaged

44120 times in Balb/c mice followed by deep sequencing which identified 3 additional acquired

442 mutations (S T417N, S H655Y, and E E8V). This virus, termed MA-SARS-CoV-2 was passaged

443 once in VeroE6 cells to generate a working stock. The B.1.351 variant was provided by Dr. Andy

444 Pekosz (John Hopkins University, Baltimore, MD) and grown on VeroE6-TMPRSS2 cells and viral

445 titers were determined by plaque assay on VeroE6 cells (ATCC). The generation of TMPRSS2-

446 hACE2 cells were previously described ${ }^{34}$. VERO E6 cells (ATCC CRL-1586) were transfected

447 with pCAGGS plasmid in which chicken actin gene promoter drives the expression of an open

448 reading frame comprising Puromycin N-acetyl transferase, GSG linker, 2A self-cleaving peptide

449 of thosea asigna virus (T2A), human transmembrane serine protease 2 (TMPRSS2). Two days

450 post-transfection, cells were trypsinzed and transferred to a $100 \mathrm{~mm}$ dish containing complete

451 DMEM medium (1x DMEM, Thermo Fisher, \# 11965118, 10\% FBS, 1x penicillin/streptomycin)

452 supplemented with puromycin (Thermo Fisher, \#A1113803) at a final concentration of $10 \mu \mathrm{g} / \mathrm{ml}$.

453 Approximately ten days later, individual colonies of cells were isolated using cloning cylinders

454 (Sigma) and expanded in medium containing puromycin. Clonal cell lines were screened for

455 expression of TMPRSS2 by flow cytometry. VeroE6-TMPRSS2 cells and VeroE6-TMPRSS2-

456 hACE2 cells were cultured in complete DMEM in the presence of Gibco Puromycin $10 \mathrm{mg} / \mathrm{mL}$ (\# 
A11138-03). VeroE6 cells were cultured in complete DMEM medium consisting of 1x DMEM

458 (Corning Cellgro), 10\% FBS, 25 mM HEPES Buffer (Corning Cellgro), 2 mM L-glutamine, 1mM

459 sodium pyruvate, 1x Non-essential Amino Acids, and 1x antibiotics.

461 Infection of mice with MA-SARS-CoV-2. C57BL/6J and Ccr2-- mice were purchased from

462 Jackson Laboratories or bred in-house at the Yerkes National Primate Research Center rodent

463 facility at Emory University. All mice used in these experiments were females between 8-12 weeks

464 of age. Stock MA-SARS-CoV-2 or B.1.351 virus was diluted in PBS to a working concentration

465 of $1 \times 10^{7} \mathrm{PFU} / \mathrm{mL}$. Mice were anesthetized with isoflurane and infected intranasally with virus (50

466 uL, $5 \times 10^{5}$ PFU/mouse) in an ABSL-3 facility. Mice were monitored daily for weight loss. All

467 experiments adhered to the guidelines approved by the Emory University Institutional Animal Care 468 and Committee.

470 Quantification of infectious virus. At the indicated day post infection, mice were euthanized

471 via isoflurane overdose and lung tissue was collected in Omni-Bead ruptor tubes filled with 1\%

472 FBS-HBSS. Tissue was homogenized in an Omni Bead Ruptor 24 (5.15 ms, 15 seconds). To

473 perform plaque assays, 10-fold dilutions of viral supernatant in serum free DMEM (VWR, \#45000-

474 304) were overlaid on VeroE6-TMPRSS2-hACE2 cells monolayers and adsorbed for 1 hour at

$47537^{\circ} \mathrm{C}$. After adsorption, $0.5 \%$ immunodiffusion Agarose in 2X DMEM supplemented with 5\% FBS

476 (Atlanta Biologics) and 1X sodium bicarbonate was overlaid, and cultures were incubated for 48

477 hours at $37^{\circ} \mathrm{C}$. Agarose plugs were removed, cells fixed with 4\% PBS-buffered paraformaldehyde

478 for 15 minutes at room temperature and plaques were visualized using crystal violet staining $(20 \%$ 479 methanol in $\mathrm{ddH}_{2} \mathrm{O}$ ). 
Quantitative reverse-transcription PCR of lung tissues. At the indicated day post infection, mice were euthanized with isoflurane overdose and one lobe of lung tissue was collected in an

483 Omni Bead ruptor tube filled with Tri Reagent (Zymo, \#R2050-1-200). Tissue was homogenized 484 using an Omni Bead Ruptor 24 (5.15 ms, 15 seconds), then centrifuged to remove debris. RNA 485 was extracted using a Direct-zol RNA MiniPrep Kit (Zymo, \# R2051), then converted to cDNA 486 using a High-capacity Reverse Transcriptase cDNA Kit (Thermo, \#4368813). RNA levels were 487 quantified using the IDT Prime Time Gene Expression Master Mix, and Taqman gene expression 488 Primer/Probe sets (IDT). All qPCR was performed in 384- well plates and run on a QuantStudio5 489 qPCR system. SARS-CoV-2 RNA-dependent RNA polymerase levels were measured as 490 previously described ${ }^{5}$. SARS-CoV-2 E protein subgenomic RNA (sgRNA) was measured using 491 the E_Sarbeco set from IDT: F1 forward primer, $100 \mathrm{nmol}$ (\#10006889), R2 reverse primer, 100 $492 \mathrm{nmol}$ (\#10006891), P1 probe, $50 \mathrm{nmol}$ (\#10006893). The following Taqman Primer/Probe sets 493 (ThermoFisher) were used in this study: Gapdh (Mm99999915_g1), Ifnl2 (Mm04204155_gH), Ifit2 494 (Mm00492606_m1), Ifih1(Mm00459183_m1), Ifnb1 (Mm00439552_s1), Ifna4 495 (Mm00833969_s1), Ccl2 (Mm00441242_m1), Ccl5 (Mm01302427_m1), Cxcl10 496 (Mm99999072_m1), Tnf (Mm00443258_m1), Il1b (Mm00434228_m1), II6 (Mm00446190_m1).

Processing of mouse tissues to single cell suspensions. At the indicated day post infection, 499 mice were anesthetized using isoflurane and injected retro-orbitally with CD45:PE (100 ul per 500 mouse, diluted 1:20 in PBS). Mice were allowed to recover for 5 minutes, then euthanized via 501 isoflurane overdose. One lobe of lung tissue and spleens were collected from each mouse and 502 placed in 1\% FBS-HBSS. Spleens were mechanically homogenized on a 70 uM cell strainer, and 503 the cell suspension was collected in 10\% FBS-RPMI. Splenocyte suspension was spun down 504 (1250 rpm, 5 min, 4C) and lysed in ACK Lysis buffer (Lonza) for 5 minutes on ice. Splenocytes 505 were washed with 10\%FBS-RPMI then kept on ice until ready for downstream applications. Lungs 
were mechanically disrupted in 6-well plates, then digested for 30 minutes at $37 \mathrm{C}$ in a solution of

507 DNasel (2000U/mL, Sigma D4527-500KU)) and collagenase (5mg/mL, (Sigma, \#11088882001)

508 in HBSS. Digestion was stopped with 10\% FBS-RPMI, and lungs were pushed through a 70 uM

509 filter to obtain a single cell suspension. Cells were resuspended in $30 \%$ Percoll-PBS and

510 centrifuged at $2000 \mathrm{rpm}$ for 20 minutes. The top layer of cell debris was removed and the cell-

511 pellet at the bottom was lysed with ACK lysis buffer for 5 minutes on ice. Cells were washed, and

512 resuspended in 10\%FBS-RPMI and kept on ice until ready for staining.

514 Flow cytometry analysis. Single-cell suspensions were spun down, and resuspended in anti-

515 CD16/32 (Tonbo, Clone 2.4G2) blocking solution for 20 minutes at $4^{\circ} \mathrm{C}$. Cell suspensions were 516 spun down, and stained with Live/Dead Ghost Dye stain (Tonbo Biosciences) for 20 minutes at $5174^{\circ} \mathrm{C}$. Cells were washed, and resuspended in the indicated surface stain in FACS buffer for 20 518 minutes at $4^{\circ} \mathrm{C}$. After staining, cells were washed and fixed in $2 \%$ PFA-PBS for 20 minutes at 519 room temperature. Precision count beads (Biolegend) were added to samples to obtain counts.

520 Samples were run on a BD FACS Symphony A5. The following antibodies were used in this study:

521 CD45:PE (Biolegend, Clone: 30-F11), CD45.2:BV605 (Biolegend, Clone: 104), CD11b:BUV395

522 BD Biosciences, 440c), I-A/I-E:AF700 (Biolegend, M5/114.15.2), CD11c:BUV737 (Biolegend,

523 N418), CD26: PE-Cy7 (Biolegend, H194-112), CD172a:BV510 (Biolegend, P84), XCR1:AF647

524 (Biolegend, Zet), CD64: PerCpCy5.5 (Biolegend, X54-5/7.1), F4/80:FITC (Biolegend, BM8),

525 H2kb:BUV805 (BD Biosciences, AF6-88.5), CD86:PE-Dazzle594 (Biolegend, GL1), Live/Dead

526 Ghost Dye:R780 (Tonbo), CD3:APC-Cy7 (Biolegend, 145-2C11), CD19:APC-Cy7 (BD

527 Biosciences, 1D3), NK1.1 (Biolegend, PK136), Ly6C:BV785 (Biolegend, Hk1.4), Ly6G:BV650

528 (Biolegend, 1A8), SiglecF:BV421 (BD Biosciences, E50-2440). 
530 In situ hybridization of lung tissues. One lobe of the lung was harvested from mice at 0 or 4

531 days p.i., and fixed with 4\% PFA-PBS for a minimum of 3 days. Formalin-fixed paraffin-embedded

532 lung tissue were deparaffinized through sequential washes twice each in xylene and $100 \%$

533 ethanol for 5 min. Tissue were then pretreated with RNAscope Hydrogen Peroxide for 10 min at

$534 \mathrm{RT}$, then RNAscope Target Retrieval for $5 \mathrm{~min}$ at $95-100{ }^{\circ} \mathrm{C}$, followed by RNAscope Protease

535 Plus for $30 \mathrm{~min}$ at $40{ }^{\circ} \mathrm{C}$. RNA-ISH was performed using a probe against the S gene of SARS-

536 CoV-2 (V-nCoV-2019-S, ACD) using the RNAscope $2.5 \mathrm{HD}$ Assay-BROWN as per the

537 manufacturer's instructions. Slides were covered slipped with ProLong Gold Antifade Mountant

538 (Thermo Fisher). Images were acquired using a Zeiss Axiolmager Z2 system with Zeiss software.

540 Single-cell RNA-Seq analysis. Lungs from mice at 0 or 4 days p.i., were processed to single

541 cells suspensions as described above. Single cell suspensions were washed 4 times with PBS,

542 and passed through a 70 uM filter. Cell suspensions were counted, and captured in droplets

543 using the Chromium NextGEM Single Cell 5' Library \& Gel Bead kits on a 10x Chromium

544 controller in a BSL-3 biosafety cabinet. Amplification of cDNA and library preparation was

545 performed according to the manufacturer's instructions. Gene expression libraries were

546 sequenced as paired-end 26x91 reads on an Illumina NovaSeq6000 targeting a depth of 50,000

547 reads per cell in the Yerkes Genomics Core Laboratory

548 (http://www.yerkes.emory.edu/nhp_genomics_core/). Analysis was conducted using R (v4) and

549 Seurat (v4). Cell Ranger (v6) was used for demultiplexing, aligning barcodes, mapping to the

550 genome (mm10) and quantifying UMls. Filtered Cell Ranger matrices were processed with

551 Read10x function in Seurat for preprocessing and cluster analysis. Data was filtered to remove

552 cells with less than 200 genes, abnormally high gene counts (feature counts $>5000$ ) and greater

553 than $5 \%$ mitochondrial genes. After qualify control, there were 9,399 mock cells and 10,982

554 CoV2 cells. Principal component analysis (PCA) and dimensional reduction was conducted on 
555 Log-normalized and scaled gene expression data. Clustering was conducted using

556 FindNeighbors and FindClusters functions, with resolution parameters between 0.5-1.4. Overall,

55723 clusters were identified and FindAllMarkers function utilized to identify DEGs, from which

558 marker genes for cluster cell annotation was conducted. After annotating cells, DEGs were

559 determined based on sub-clusters or experimental group. Gene set enrichment analysis (GSEA)

560 was conducted using ranked gene list produced with Seurat FindMarkers function (comparing

561 CoV2 samples with mock samples) and Genelist were obtained from: MsigDB (hallmarks) and

562 https://www.nature.com/articles/s41422-020-00455-9\#MOESM1 (macrophage suppressive and

563 hyperinflammatory). GSEA was conducted using Broad (4.0.3) and plotted in R.

564

565 Data availability

566 Single cell RNA sequencing data will be publicly accessible through Gene Expression Omnibus

567 following acceptance of this article. 


\section{Acknowledgements}

569 This work was supported in part by grants (P51 OD011132 and R56 Al147623 to Emory 570 University) from the National Institute of Allergy and Infectious Diseases, National Institutes of

571 Health, by intramural funding from the National Institute of Allergy and Infectious Diseases, the

572 Emory Executive Vice President for Health Affairs Synergy Fund award, the Pediatric Research

573 Alliance Center for Childhood Infections and Vaccines and Children's Healthcare of Atlanta, the

574 Emory-UGA Center of Excellence for Influenza Research and Surveillance, and Woodruff Health

575 Sciences Center 2020 COVID-19 CURE Award. J.T. is supported by T32 AI074492. We thank

576 Andy Pekosz for the B.1.351 variant. Next-generation sequencing services were provided by the

577 Yerkes NHP Genomics Core, which is supported in part by NIH P51 OD 011132, and the data

578 were acquired on a NovaSeq 6000 funded by NIH S10 OD 026799. We also thank Eli Boritz and

579 Danny Douek for sequencing and analysis of the B.1.351 variant (NIAID/NIH, Atlanta, GA).

\section{Author Contributions}

582 A.V. and M.S.S. contributed to the acquisition, analysis, and interpretation of the data, as well as

583 the conception and design of the work, and writing the manuscript. J.T. and J.K. contributed to

584 the analysis and interpretation of the data, and writing the manuscript. A.S. contributed to the 585 acquisition, analysis, and interpretation of the data, and writing the manuscript. M.D.G, K.F., A.C, 586 A.P, A.D-C, and F.J. contributed to the acquisition and analysis of the data. R.K. contributed to 587 the analysis, interpretation of the data, and conception and design of the work. S.E.B., D.A.C, 588 K.P., A.G., P.S. and V.D.M. contributed to the interpretation of the data and conception and design 589 of the work. 


\section{References}

5931 Zhou, P. et al. A pneumonia outbreak associated with a new coronavirus of probable bat 594 origin. Nature 579, 270-273, doi:10.1038/s41586-020-2012-7 (2020).

5952 Zhu, N. et al. A Novel Coronavirus from Patients with Pneumonia in China, 2019. N Engl

$596 \quad$ J Med 382, 727-733, doi:10.1056/NEJMoa2001017 (2020).

5973 Yin, X. et al. MDA5 Governs the Innate Immune Response to SARS-CoV-2 in Lung

$598 \quad$ Epithelial Cells. Cell reports 34, 108628, doi:10.1016/j.celrep.2020.108628 (2021).

5994 Arunachalam, P. S. et al. Systems biological assessment of immunity to mild versus

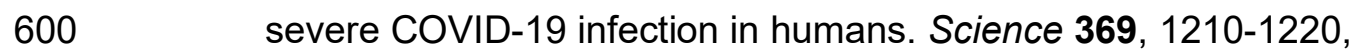

601 doi:10.1126/science.abc6261 (2020).

6025 Vanderheiden, A. et al. Type I and Type III Interferons Restrict SARS-CoV-2 Infection of 603 Human Airway Epithelial Cultures. Journal of virology 94, doi:10.1128/jvi.00985-20

604 (2020).

6056 Liao, M. et al. Single-cell landscape of bronchoalveolar immune cells in patients with

606 COVID-19. Nature Medicine 26, 842-844, doi:10.1038/s41591-020-0901-9 (2020).

6077 Wang, C. et al. Alveolar macrophage dysfunction and cytokine storm in the

608 pathogenesis of two severe COVID-19 patients. EBioMedicine 57,

609 doi:10.1016/j.ebiom.2020.102833 (2020).

6108 Szabo, P. A. et al. Longitudinal profiling of respiratory and systemic immune responses

611 reveals myeloid cell-driven lung inflammation in severe COVID-19. Immunity,

612 doi:https://doi.org/10.1016/j.immuni.2021.03.005 (2021).

6139 Geissmann, F., Jung, S. \& Littman, D. R. Blood monocytes consist of two principal

614 subsets with distinct migratory properties. Immunity 19, 71-82, doi:10.1016/s1074-

615 7613(03)00174-2(2003). 
61610 Srivastava, M. et al. The Inflammatory versus Constitutive Trafficking of Mononuclear

617 Phagocytes into the Alveolar Space of Mice Is Associated with Drastic Changes in Their

618 Gene Expression Profiles. The Journal of Immunology 175, 1884,

619 doi:10.4049/jimmunol.175.3.1884 (2005).

62011 Lin, K. L., Suzuki, Y., Nakano, H., Ramsburg, E. \& Gunn, M. D. CCR2+ monocyte-

621 derived dendritic cells and exudate macrophages produce influenza-induced pulmonary

622 immune pathology and mortality. Journal of immunology (Baltimore, Md. : 1950) 180,

623 2562-2572, doi:10.4049/jimmunol.180.4.2562 (2008).

62412 Seo, S. U. et al. Type I interferon signaling regulates Ly6C(hi) monocytes and

625 neutrophils during acute viral pneumonia in mice. PLoS Pathog 7, e1001304,

626 doi:10.1371/journal.ppat.1001304 (2011).

62713 Cao, W. et al. Rapid differentiation of monocytes into type I IFN-producing myeloid

628 dendritic cells as an antiviral strategy against influenza virus infection. Journal of

629 immunology (Baltimore, Md. : 1950) 189, 2257-2265, doi:10.4049/jimmunol.1200168

$630 \quad$ (2012).

63114 Xie, X. et al. An Infectious cDNA Clone of SARS-CoV-2. Cell Host Microbe 27, 841-848

632 e843, doi:10.1016/j.chom.2020.04.004 (2020).

$63315 \mathrm{Gu}, \mathrm{H}$. et al. Adaptation of SARS-CoV-2 in BALB/c mice for testing vaccine efficacy.

634 Science 369, 1603-1607, doi:10.1126/science.abc4730 (2020).

63516 Hou, Y. J. et al. SARS-CoV-2 Reverse Genetics Reveals a Variable Infection Gradient in

636 the Respiratory Tract. Cell, doi:https://doi.org/10.1016/j.cell.2020.05.042 (2020).

63717 Tegally, H. et al. Detection of a SARS-CoV-2 variant of concern in South Africa. Nature

$638 \quad 592,438-443$, doi:10.1038/s41586-021-03402-9 (2021).

63918 Guo, Q. et al. Induction of alarmin S100A8/A9 mediates activation of aberrant

640 neutrophils in the pathogenesis of COVID-19. Cell Host \& Microbe 29, 222-235.e224,

641 doi:https://doi.org/10.1016/i.chom.2020.12.016 (2021). 
64219 Tsou, C.-L. et al. Critical roles for CCR2 and MCP-3 in monocyte mobilization from bone 643 marrow and recruitment to inflammatory sites. The Journal of Clinical Investigation 117, $644 \quad$ 902-909, doi:10.1172/JCl29919 (2007).

64520 Chua, R. L. et al. COVID-19 severity correlates with airway epithelium-immune cell 646 interactions identified by single-cell analysis. Nature Biotechnology 38, 970-979, 647 doi:10.1038/s41587-020-0602-4 (2020).

64821 Schulte-Schrepping, J. et al. Severe COVID-19 Is Marked by a Dysregulated Myeloid 649 Cell Compartment. Cell 182, 1419-1440.e1423, doi:10.1016/j.cell.2020.08.001 (2020).

65022 Silvin, A. et al. Elevated Calprotectin and Abnormal Myeloid Cell Subsets Discriminate 651 Severe from Mild COVID-19. Cell 182, 1401-1418.e1418, doi:10.1016/j.cell.2020.08.002 652 (2020).

65323 Xiong, Y. et al. Transcriptomic characteristics of bronchoalveolar lavage fluid and peripheral blood mononuclear cells in COVID-19 patients. Emerg Microbes Infect 9, 761770, doi:10.1080/22221751.2020.1747363 (2020).

24 Fajnzylber, J. et al. SARS-CoV-2 viral load is associated with increased disease severity and mortality. Nature Communications 11, 5493, doi:10.1038/s41467-020-19057-5 (2020).

65925 Peters, W. et al. Chemokine receptor 2 serves an early and essential role in resistance to Mycobacterium tuberculosis. Proc Natl Acad Sci U S A 98, 7958-7963, doi:10.1073/pnas.131207398 (2001).

66226 Roy, R. M., Wüthrich, M. \& Klein, B. S. J. T. J. o. I. Chitin elicits CCL2 from airway 663 epithelial cells and induces CCR2-dependent innate allergic inflammation in the lung.

$664 \quad 189,2545-2552(2012)$.

66527 Liegeois, M., Legrand, C., Desmet, C. J., Marichal, T. \& Bureau, F. The interstitial 666 macrophage: A long-neglected piece in the puzzle of lung immunity. Cellular 667 immunology 330, 91-96, doi:10.1016/j.cellimm.2018.02.001 (2018). 
66828 Guilliams, M. et al. Alveolar macrophages develop from fetal monocytes that differentiate 669 into long-lived cells in the first week of life via GM-CSF. The Journal of experimental 670 medicine 210, 1977-1992, doi:10.1084/jem.20131199 (2013).

67129 Hashimoto, D. et al. Tissue-resident macrophages self-maintain locally throughout adult 672 life with minimal contribution from circulating monocytes. Immunity 38, 792-804, 673 doi:10.1016/j.immuni.2013.04.004 (2013).

$67430 \quad$ Yona, S. et al. Fate mapping reveals origins and dynamics of monocytes and tissue 675 macrophages under homeostasis. Immunity 38, 79-91, 676 doi:10.1016/j.immuni.2012.12.001 (2013).

67731 Nakano, H., Lyons-Cohen, M. R., Whitehead, G. S., Nakano, K. \& Cook, D. N. Distinct 678 functions of CXCR4, CCR2, and CX3CR1 direct dendritic cell precursors from the bone 679 marrow to the lung. J Leukoc Biol 101, 1143-1153, doi:10.1189/jlb.1A0616-285R (2017).

68032 Herold, S. et al. Lung epithelial apoptosis in influenza virus pneumonia: the role of 681 macrophage-expressed TNF-related apoptosis-inducing ligand. The Journal of 682 experimental medicine 205, 3065-3077, doi:10.1084/jem.20080201 (2008).

68333 Grant, R. A. et al. Circuits between infected macrophages and T cells in SARS-CoV-2 684 pneumonia. Nature, doi:10.1038/s41586-020-03148-w (2021).

68534 Chen, R. E. et al. Resistance of SARS-CoV-2 variants to neutralization by monoclonal 686 and serum-derived polyclonal antibodies. Nat Med, doi:10.1038/s41591-021-01294-w $687 \quad(2021)$ 